\title{
Open questions in turbulent stratified mixing: Do we even know what we do not know?
}

\author{
Colm-cille P. Caulfield $\odot^{*}$ \\ BP Institute, University of Cambridge, Cambridge CB3 OEZ, United Kingdom \\ and Department of Applied Mathematics and Theoretical Physics, University of Cambridge, \\ Cambridge CB3 OWA, United Kingdom
}

(Received 7 August 2020; accepted 20 October 2020; published 24 November 2020)

\begin{abstract}
Understanding how turbulence leads to the enhanced irreversible transport of heat and other scalars (such as salt and pollutants) in density-stratified fluids is a fundamental and central problem in geophysical and environmental fluid dynamics. There is a wide range of highly important applications, an important example being the description and parameterization of diapycnal transport in the world's oceans, a key area of uncertainty in climate modeling. Recently, possibly due to the proliferation of data obtained through direct observation, numerical simulation, and laboratory experimentation, there has been an explosion in research activity directed at improving community understanding, modeling, and parametrization of the subtle interplay among energy conversion pathways, turbulence, and irreversible mixing in density-stratified fluids. However, there are still leading-order open questions and areas of profound uncertainty concerning this interesting and important research challenge.
\end{abstract}

DOI: 10.1103/PhysRevFluids.5.110518

\section{INTRODUCTION}

This paper summarizes certain aspects of the invited talk (with the same title) that I gave on Sunday November 24, 2019, as part of the 72nd Annual Meeting of the APS Division of Fluid Dynamics, held in Seattle, WA. It presents my personal perspective on some priorities for further research into the hugely complex, important and fascinating challenge of irreversible turbulent mixing in stratified fluids. In this paper, I attempt to articulate my personal, indeed idiosyncratic, view of turbulent mixing in stratified fluids, with a particular focus on various fundamental fluid-dynamical aspects of ongoing uncertainty and controversy. Parameterizing such mixing is a key component of larger-scale models of the world's oceans [1], where there are of course a huge range of different processes which lead to small-scale mixing, not least associated with the interaction between topography, internal waves, and tidal motions [2]. However, here I focus on highly idealized flow configurations that I have considered with a large number of collaborators. Each flow configuration has specific practical attractions due to various computational and modeling features. My personal objective in this paper is not to present a conventional review of the present state of even just the fluid-dynamical community's research into the important, and indeed pressing, climate-crisis-relevant challenge of parametrizing turbulent stratified mixing [3]. The overarching motivation is to contribute insights useful for the improvement of such parametrization, with a particular aim of application to modeling ocean mixing. Nevertheless, at least in this paper, I do not impose practical constraints concerning what actually has been (or indeed can conceivably be) measured or observed in real flows. I believe it is important to understand (as much as possible)

*cpc12@cam.ac.uk 
turbulent stratified mixing when there is access to all relevant data and from that data then identify models which might be constructed to be consistent with and useful for real-world applications.

Put simply, the "end" here is to understand as much as possible about the causes and properties of turbulent stratified mixing in and of itself, rather than viewing such an understanding as a "means" toward a broader, yet inevitably more applied "end" of geophysical, environmental, or indeed industrial fluid systems. Therefore, my objective in this particular paper is to achieve two aims. First, I wish to demonstrate the subtle (and often counterintuitive) complexities of the energetics of stratified mixing through consideration of a small number of illustrative examples. Second, I wish to use results and insights arising from these examples to motivate further research in this fascinating and challenging area.

To address these twin and interconnected aims, the rest of this paper is organized as follows. In Sec. II, I discuss in detail certain key aspects of the energetics of stratified turbulent flows, drawing out the central role played by an appropriate definition of the "(turbulent) flux coefficient" $\Gamma$, essentially the rate at which turbulent dissipation is "taxed" by the stratification. In Sec. III, apparently conflicting evidence is presented concerning the dependence of flux coefficients $\Gamma$ (defined in various ways) on the "buoyancy Reynolds number," in body- or wall-forced shear flows, which forcing ensures an approach to quasistationarity. The properties of such quasistationary forced flows are then contrasted in Sec. IV with the properties of freely evolving shear flows prone to primary vortical instabilities. As I argue below, in both these situations, it is reasonable to characterize the flows in as being "weakly stratified," in the loose sense that the mixing of the scalar density (ultimately through diffusion) is "slaved" to the largely unaffected mixing of momentum (through the enhanced viscous dissipation). Motivated by the desire to see if it is possible to have vigorous mixing in flows which might be characterized as being "strongly stratified," in Sec. V, forced flows which are specifically designed in an attempt to access more strongly stratified flow regimes are considered. Here the apparently generic spontaneous appearance of "layering" is demonstrated, i.e., the organization of the flow into regions of relatively "strong" and "weak" stratification. Tantalizing, but as yet undoubtedly inconclusive, evidence is presented that energetic turbulence in "strongly" stratified flows is inevitably associated with spatiotemporally intermittent layers where locally the stratification should still be characterized as being "weak" in some sense. The implications and potential applications of this evidence, as well as signposts pointing toward some of the most interesting open questions, are discussed in Sec. VI.

\section{HOW SHOULD STRATIFIED MIXING ENERGETICS BE QUANTIFIED?}

Here attention is restricted to the idealized situation of a "Boussinesq" fluid with a linear (imposed) equation of state. The fluid density $\rho(\boldsymbol{x}, t)$ is assumed to vary relative to a reference value $\rho_{r}$ by sufficiently small amounts so that $\left|\rho(\boldsymbol{x}, t)-\rho_{r}\right| \ll \rho_{r}$ and such variations are only significant in the buoyancy force. Furthermore, density variations only depend on a single scalar field (for example, the fluid's temperature), which crucially is diffusive with (molecular) diffusivity $\kappa$. The fluid velocity field $\boldsymbol{u}(\boldsymbol{x}, t)$ is assumed to be divergence free $\boldsymbol{\nabla} \cdot \boldsymbol{u}=0$. Therefore, the pressure field is not associated with thermodynamic properties of the fluid, but rather the pressure is effectively a nonlocal Lagrange multiplier imposing the constraint of divergence-free velocity field, i.e., that the fluid is "incompressible."

These assumptions have several strong consequences. Important real-world processes, such as double-diffusion associated with the dependence of the density on two scalars with two different diffusivities [4] or compressibility, thermodynamics, viscous heating, etc. [5,6], are (deliberately) suppressed. Nevertheless, the dynamical behavior of this simplified system is still very complex and rich. An important way to see this is to consider the "turbulent" kinetic energy equation, arising if the velocity and density fields are decomposed into a "mean" and a fluctuation or "turbulent" component using a Reynolds decomposition:

$$
\boldsymbol{u}=\langle\boldsymbol{u}\rangle+\boldsymbol{u}^{\prime}=\boldsymbol{U}+\boldsymbol{u}^{\prime} ; \quad \rho=\langle\rho\rangle+\rho^{\prime}=\bar{\rho}+\rho^{\prime},
$$


where the angle brackets denote an appropriate averaging (perhaps formally most elegantly an ensemble average but in practice often a spatial and/or temporal average).

\section{A. Turbulent kinetic energy}

In many circumstances of interest, there is some mean shear, due, for example, to wall-forcing, artificially introduced forcing terms in a numerical simulation or even set in an initial value problem which is allowed to evolve freely subsequently. Making the further assumptions for such shear flows that the mean velocity $\mathbf{U}=\hat{\mathbf{x}}_{1} U\left(x_{3}, t\right)$, using the geophysical coordinate system where gravity acts in the (negative) $x_{3}$ direction, the evolution equation for the turbulent kinetic energy $\mathcal{K}^{\prime}$ in a Boussinesq fluid can be written symbolically (using the Einstein summation convention) as

$$
\frac{1}{2} \frac{\partial}{\partial t}\left\langle u_{i}^{\prime} u_{i}^{\prime}\right\rangle \equiv \frac{\partial}{\partial t} \mathcal{K}^{\prime}=-\mathcal{E}-\mathcal{B}+\mathcal{P}+\mathcal{F}-\nabla \cdot \mathcal{J}
$$

On the right-hand side of this equation, the divergence of the flux $\mathcal{J}$ captures various transport and redistribution processes within the domain of interest, associated with viscous-, pressure-, and advection-driven processes. With appropriate boundary conditions on the domain of interest (and application of the divergence theorem), this term can often be ignored or alternatively be quantified directly through boundary contributions. In a domain with moving boundaries (e.g., plane Couette flow between two parallel infinite plates moving relative to each other) such boundary contributions can force the turbulent flow.

Furthermore, $\mathcal{F}$ represents a body-forcing, as is often imposed in numerical simulations to represent some unresolved physical process which injects energy right into the turbulent velocity field, in some sense artificially.

The other three terms, the turbulence production term $\mathcal{P}$, the "buoyancy" flux $\mathcal{B}$ (which with the sign convention used here should more appropriately be called the density flux), and the (turbulent) dissipation rate $\mathcal{E}$ are

$$
\mathcal{P} \equiv-\left\langle u_{1}^{\prime} u_{3}^{\prime}\right\rangle \frac{\partial}{\partial x_{3}} U ; \quad \mathcal{B} \equiv \frac{g}{\rho_{r}}\left\langle u_{3}^{\prime} \rho^{\prime}\right\rangle ; \quad \mathcal{E} \equiv 2 v\left\langle s_{i j}^{\prime} s_{i j}^{\prime}\right\rangle ; \quad s_{i j}^{\prime} \equiv \frac{1}{2}\left(\frac{\partial u_{i}^{\prime}}{\partial x_{j}}+\frac{\partial u_{j}^{\prime}}{\partial x_{i}}\right),
$$

where $s_{i j}^{\prime}$ is the (perturbation velocity) gradient tensor.

The turbulent kinetic energy $\mathcal{K}^{\prime}$ can also grow through extracting energy from any mean shear via the Reynolds stress and mean shear $S \equiv \partial U / \partial x_{3}$ in the turbulence production term $\mathcal{P}$, which could also be maintained by some (imposed) forcing, either through boundary contributions or (analogously to $\mathcal{F}$ ) through extra terms artificially added in a numerical simulation to model some unresolved process.

By whatever mechanism it occurs, such a tendency for $\mathcal{K}^{\prime}$ to increase in the domain of interest (and to be transported around the domain through the action of $\mathcal{J}$ ) is balanced by the other terms. The positive-definite dissipation rate $\mathcal{E}$ extracts kinetic energy irreversibly, and such dissipation is markedly increased in turbulent flow (indeed this might be argued to be a defining characteristic of "turbulence") due to the proliferation of high gradients within such flows. It is self-evident that understanding and modeling turbulent flows is a profoundly challenging problem of great theoretical and practical interest to mathematicians, scientists, and engineers. This challenge is made even harder by the added complexity associated with the density flux term $\mathcal{B}$, which generically is nonzero in stratified flows.

This term captures the conversion of kinetic energy to and from potential energy, as $\mathcal{B}>0$ occurs when $u_{3}^{\prime}$ and $\rho^{\prime}$ are (positively) correlated, and so, on average (relatively), dense parcels are being lifted up within a gravitational field. It also may be thought of as the rate at which stratification "taxes" turbulence. For every unit of energy injected through one of the three forcings described above (i.e., body forcing, boundary contributions, or turbulent shear production) generically some of it is viscously dissipated (via $\mathcal{E}$ ) and some is converted to potential energy (via $\mathcal{B}$ ). Indeed, 
modeling and parametrizing this "taxation" rate has been a major area of research (and controversy) for decades [7-9].

\section{B. Mixing parameters}

Two classic nondimensional parameters which have been considered to quantify this taxation rate are the "flux Richardson number" $\mathrm{Ri}_{f}$ and the "turbulent flux coefficient" $\Gamma$ (sometimes, somewhat confusingly, called "mixing efficiency") which may be defined as ratios of particular terms in (2)

$$
\mathrm{Ri}_{f} \equiv \frac{\mathcal{B}}{\mathcal{P}} ; \quad \Gamma_{\mathcal{B}} \equiv \frac{\mathcal{B}}{\mathcal{E}},
$$

where the subscript $\mathcal{B}$ has been added to make the particular dependence on $\mathcal{B}$ explicit. $\Gamma_{\mathcal{B}}$ (defined in this way) was first introduced in a deeply influential paper by Osborn [10], where further assumptions were made that the turbulence was in a statistically steady state and the various transport terms could be ignored, thus leading to a simple relationship $\mathrm{Ri}_{f}=\Gamma_{\mathcal{B}} /\left(1+\Gamma_{\mathcal{B}}\right)$. Furthermore, from experimental evidence, Osborn argued that $\Gamma_{\mathcal{B}} \leqslant 0.2$, though often in the oceanographic literature in particular [11], equality is assumed.

Perhaps the biggest reason why $\Gamma_{\mathcal{B}}$ has attracted so much attention is that it naturally arises within the definition of a turbulent or eddy diffusivity for heat (assuming that heat is the stratifying agent):

$$
\kappa_{T} \equiv \frac{\frac{g}{\rho_{r}}\left\langle u^{\prime}{ }_{3} \rho^{\prime}\right\rangle}{\frac{g}{\rho_{r}}\left|\partial \bar{\rho} / \partial x_{3}\right|} \equiv \frac{\mathcal{B}}{N^{2}},
$$

where $N$ is the buoyancy frequency, which in general it must be remembered, could be a function of space and/or time. Using the definition for $\Gamma_{\mathcal{B}}, \kappa_{T}$ can thus be expressed as

$$
\kappa_{T}=\Gamma_{\mathcal{B}} \frac{\mathcal{E}}{N^{2}}=v \Gamma_{\mathcal{B}}\left(\frac{\mathcal{E}}{v N^{2}}\right) \equiv v \Gamma_{\mathcal{B}} \operatorname{Re}_{b},
$$

where $v$ is the (molecular) kinematic viscosity and $\operatorname{Re}_{b}$ is (commonly) referred to as the "buoyancy Reynolds number." This expression is very appealing, suggesting a natural way to construct $\kappa_{T}$, although it is always important to remember that (4) and (5) are specific definitions that inevitably lead to (6) without any added insight. Indeed, since $\kappa_{T}$ parameterizes the density flux as a (turbulent) diffusive process, it (at least implicitly) connects the density flux $\mathcal{B}$ to the (turbulent) mixing of the density field. However, there are (at least) two obvious criticisms of this approach.

\section{Criticism 1: Reversibility}

The first criticism is that the density flux is not sign definite and actually quantifies the (in general) two-way exchange between the potential and kinetic energy reservoirs. If the flow on average pushes dense parcels down, as naturally happens during "convection," then $\mathcal{B}<0$, and potential energy is actually converted into kinetic energy. Any observation of convection or the mixing induced by Rayleigh-Taylor instability (see, e.g., [12]) reveals immediately that such convectively driven mixing can be very vigorous. Applying ideas originally due to Lorenz [13-15] convincing arguments have been presented $[14,15]$ to identify irreversible mixing with changes to the "background" potential energy, i.e., the minimum potential energy of the system accessible by volume-conserving resorting of fluid elements.

The potential energy of the system can be thought to be divided into two parts, this background potential energy and the remaining "available" potential energy, which can drive fluid motions. Generically, the density flux can then be thought to be the sum of two terms $\mathcal{B}=\mathcal{S}+\mathcal{M}$, a sign-indefinite "stirring" rate $\mathcal{S}$ quantifying reversible exchanges between the kinetic energy and potential energy reservoirs, and the sign-definite mixing rate $\mathcal{M} \geqslant 0 . \mathcal{M}$ can be thought of as quantifying the rate at which irreversible mixing processes inherently associated with fluid motions 
increases the background potential energy and hence captures the (eventual) irreversible conversion of kinetic energy into potential energy. This rate does thus capture the "taxation" rate of the stratification on the evolving turbulent motion.

It can be argued [16] that it is more appropriate to define an "irreversible" flux coefficient $\Gamma_{\mathcal{M}}$, or even associated instantaneous $\left(\eta_{i}\right)$ and cumulative mixing efficiencies $\eta_{c}[17,18]$ :

$$
\Gamma_{\mathcal{M}} \equiv \frac{\mathcal{M}}{\mathcal{E}} ; \quad \eta_{i} \equiv \frac{\mathcal{M}}{\mathcal{M}+\mathcal{E}}=\frac{\Gamma_{\mathcal{M}}}{1+\Gamma_{\mathcal{M}}} ; \quad \Gamma_{c}(t) \equiv \frac{\int_{t_{0}}^{t} \mathcal{M} d t}{\int_{t_{0}}^{t} \mathcal{E} d t} ; \quad \eta_{c} \equiv \frac{\Gamma_{c}}{1+\Gamma_{c}} ;
$$

over some mixing event starting at time $t_{0}$. These efficiencies are guaranteed to be in the range [0,1], and also clearly have a connection with the flux Richardson number $\mathrm{Ri}_{f}$ as defined in (4), and may be thought of as capturing fundamentally the irreversible component of the exchanges between the potential energy and kinetic energy reservoirs.

\section{Criticism 2: Connection with diffusion}

However, even once reversible stirring is filtered in this way, there is still a remaining criticism that (irreversible) mixing should at its heart be considered an inherently diffusive process that homogenizes the scalar distribution, and the effects of such homogenization (or equivalently the reduction of the variance) on exchanges between different energy reservoirs (in particular into potential energy) should really be thought of as a consequence of mixing, rather than the defining characteristic of the mixing. This key point has indeed been captured by the classic approach [14,15] in that, for the simplest case of a closed system of volume $V_{0}$, the background potential energy $\mathcal{K}_{P b}$ increases monotonically at a rate $\Phi_{d}$,

$$
V_{0} \frac{d}{d t} \mathcal{K}_{P b}=\Phi_{d} \equiv \kappa \rho_{r} \int_{V}\left[\frac{|\nabla \rho|}{\left|d x_{3 *} / d \rho\right|^{-1}}\right]^{2} N_{*}^{2} d V ; \quad N_{*}^{2}=\frac{g}{\rho_{r}}\left[\frac{d x_{3 *}}{d \rho}\right]^{-1},
$$

where the molecular diffusivity of the density scalar $\kappa$ appears explicitly. In this expression, $N_{*}$ is the notional buoyancy frequency associated with the sorted density field (with minimal potential energy) with the individual fluid elements sorted to be located at $x_{3 *}$.

Generically, in a statically stable density distribution, unless $N$ is constant absolutely everywhere, even in the absence of fluid motions $\mathcal{K}_{P b}$ will increase due to the action of diffusion at a rate $\Phi_{i}$, so that $\Phi_{d}=\mathcal{M}+\Phi_{i}$. Therefore, although $\mathcal{M}$ has an implicit relationship with a diffusive mixing process, the explicit connection can be muddied due to a priori uncertainty in the relative size of $\phi_{i}$ and $\mathcal{M}$.

\section{Buoyancy variance}

Perhaps a more natural connection can be made with the evolution of an appropriately scaled "buoyancy variance." Commonly, the negative of the reduced gravity $g^{\prime}$ is referred to as the buoyancy in this context, i.e., $b \equiv-g \rho^{\prime} / \rho_{r}$. Multiplying the natural density advection-diffusion equation by $g^{2} \rho^{\prime} / \rho_{r}^{2}$ and averaging leads to

$$
\begin{aligned}
\frac{1}{N^{2}} \frac{\partial}{\partial t}\left\langle\frac{b^{2}}{2}\right\rangle & =\frac{g}{\rho_{r}}\left\langle\rho^{\prime} x_{3}^{\prime}\right\rangle-\frac{\kappa}{N^{2}}\left\langle\frac{\partial b}{\partial x_{i}} \frac{\partial b}{\partial x_{i}}\right\rangle-\frac{1}{N^{2}} \frac{\partial}{\partial x_{i}}\left[U_{i}\left\langle\frac{b^{2}}{2}\right\rangle+\left\langle\frac{u_{i}^{\prime} b^{2}}{2}\right\rangle-\kappa\left\langle b \frac{\partial b}{\partial x_{i}}\right\rangle\right], \\
\frac{1}{N^{2}} \frac{\partial}{\partial t}\left\langle\frac{b^{2}}{2}\right\rangle & =\mathcal{B}-\chi-\nabla \cdot J_{\rho} ; N^{2}\left(x_{3}, t\right) \equiv-\frac{g}{\rho_{r}} \frac{\partial}{\partial x_{3}}\langle\rho\rangle\left(x_{3}, t\right),
\end{aligned}
$$

defining an appropriately scaled destruction rate of buoyancy variance $\chi$, transport flux $J_{\rho}$, and buoyancy frequency $N$ associated with the (ensemble-averaged) density, implicitly assumed here to be a function only of $x_{3}$ and (possibly) $t$.

When formulated in this way, it is now apparent that if the transport terms have no net effect, the destruction rate of buoyancy variance $\chi$ is in lock-step with the density flux $\mathcal{B}$. However, $\chi$ has 
several inherent attractions as a measure to quantify the properties of mixing. Clearly, it maintains an explicit connection to the central role diffusion must play in (irreversible) mixing as opposed to stirring, a key concept in "passive" scalar mixing [19]. Analogously to the turbulent dissipation rate $\mathcal{E}, \chi$ is both guaranteed to be nonnegative, and can also be defined entirely locally (by making the averaging volume infinitesimal and instantaneous, for example) as opposed to the mixing rate $\mathcal{M}$ which is defined in terms of the evolution of the globally sorted density field associated with the background potential energy. Determination of such a globally sorted density field is clearly computationally expensive in numerical simulations, and in experiments or observations practically unfeasible, giving further attraction to the use of $\chi$ to quantify mixing.

Perhaps most interestingly, $\chi$ does indeed have strong connections to conversion rates of available potential energy, although it is exceptionally important to be clear on the actual definition of the buoyancy frequency which is being used, as well as the decomposition into "perturbation" and "mean" [6,20]. By comparison between (8) and (4), the key difference is that $\chi$ is normalized with the buoyancy frequency $N$ associated with the (ensemble-averaged) density, while $\Phi_{d}$ is scaled with the sorted buoyancy frequency $N_{\star}$. Indeed, if the perturbation density $\rho^{\prime}$ (and hence "buoyancy") is defined relative to and scaled with a constant (in particular with respect to $x_{3}$ and $t$ ) buoyancy frequency, then the buoyancy variance can be identified as a perturbation available potential energy, and thus $\chi$ is the destruction rate of (such an) available potential energy [6,20]. Indeed, understanding of such a relationship dates back (at least) to the early 1980s [21,22] and has been used more recently formulate an appropriate local measure of available potential energy in an attempt to quantify mixing locally [23].

With all these concepts appreciated, such an appropriately scaled destruction rate of buoyancy variance $\chi$ can thus be considered a more "natural" measure for the quantification of the rate at which stratification taxes turbulence, and so a third possible definition of the flux coefficient would be

$$
\Gamma_{\chi} \equiv \frac{\chi}{\mathcal{E}}
$$

It is becoming accepted that such a proliferation of different definitions of mixing quantities should be discouraged within the research community [1], as it proves extremely difficult to compare results from different studies. Indeed, it is well known in the passive scalar mixing community [24] that the destruction rate of scalar variance and the turbulent dissipation rate are by no means perfectly correlated, and it is somewhat curious that more sophisticated insights from passive scalar turbulent mixing have not been widely applied to the problem of parametrizing stratified mixing.

Indeed, it is not immediately apparent that $\Gamma_{\chi}$ (or equivalent mixing efficiencies) needs to be considered at all. After all, these are intermediate quantities, arising from attempting to identify relationships between different terms in the turbulent kinetic energy equation. As originally effectively argued by Osborn and Cox [25], and subsequently followed up by many authors [16,26], if the fundamental challenge is to determine the eddy diffusivity $\kappa_{T}$, using (9) removes any need to make any assumptions about the properties of the turbulent kinetic energy evolution. Remembering that the chosen definition for the eddy diffusivity in (5) has $\mathcal{B}$ in the numerator, if the "lock-step" between $\chi$ and $\mathcal{B}$ is assumed to occur to some extent, then the eddy diffusivity as defined in (5) can be approximated as

$$
\kappa_{T} \simeq \frac{\chi}{N^{2}}
$$

Indeed there is some evidence that such an "Osborn-Cox"-based approach can lead to more reliable estimates for $\kappa_{T}[16,26,27]$, suggesting that it might well be more appropriate to consider the righthand side of (11) as the underlying definition of the eddy diffusivity, without involving the density flux, and especially not its relationship to the turbulent dissipation rate via a flux coefficient. 


\section{The parametrization challenge}

There are clearly many reasonable and strong criticisms of using such blunt instruments as fluxgradient theories (leading to eddy diffusivities) to describe turbulent stratified mixing. Nevertheless, many larger-scale models rely implicitly on the use of such eddy diffusivities. Therefore, at least at the moment, the fundamental challenge to fluid dynamicists remains the modeling of $\kappa_{T}$, and in particular the dependence of $\kappa_{T}$ on various nondimensional parameters. Returning to (5), the turbulent enhancement of diffusivity is given by

$$
\frac{\kappa_{T}}{\kappa}=\Gamma_{\mathcal{B}} \operatorname{PrRe}_{b} ; \quad \operatorname{Pr} \equiv \frac{\nu}{\kappa},
$$

where Pr is the (molecular) Prandtl number. As discussed above, there are various arguments justifying other definitions than (4) for $\Gamma_{\mathcal{B}}$ for the "flux coefficient" in this expression as being more appropriate mixing measures. Indeed, there is suggestive evidence from numerical data $[16,28]$ that defining $\kappa_{T}^{*}$ [or equivalently $\operatorname{Re}_{b}^{*} \equiv \mathcal{E} /\left(v N_{*}^{2}\right)$ ] using the sorted buoyancy frequency is a more natural way to model the eddy diffusivity of heat, although as already noted, use of such globally sorted data is really only possible for simulations.

Ignoring such potentially important subtleties, the key challenge of modeling $\kappa_{T}$ is mathematically equivalent to modeling the flux coefficient. Indeed, much of the focus in research has been devoted to identifying whether the flux coefficient depends on various nondimensional parameters. From the expression (12), it is clearly important to investigate whether the flux coefficient depends on $\mathrm{Pr}$ and/or $\mathrm{Re}_{b}$, but these are by no means the only parameters which could be significant. $\mathrm{Pr}$ is an intrinsic property of the fluid, while $\mathrm{Re}_{b}$ is essentially a hybrid parameter, capturing the "intensity" of turbulence relative to the stabilizing effects of both the fluid viscosity and the background density distribution (via the buoyancy frequency $N$ ). $\operatorname{Re}_{b}$ can also be interpreted as a measure of the "dynamic range" [29] of the turbulence which can be expected to be largely unaffected by the stratification, as $\operatorname{Re}_{b}$ can be written as

$$
\operatorname{Re}_{b}=\left(\frac{L_{O}}{L_{K}}\right)^{4 / 3} ; \quad L_{O} \equiv\left(\frac{\mathcal{E}}{N^{3}}\right)^{1 / 2}, \quad L_{K} \equiv\left(\frac{\nu^{3}}{\mathcal{E}}\right)^{1 / 4},
$$

where $L_{K}$ is the Kolmogorov microscale and $L_{O}$ is the Ozmidov scale, the largest (vertical) scale which can be assumed to be largely unaffected by a background stratification. Furthermore, focusing exclusively on the properties of the flux coefficient runs the risk of obscuring the underlying dependence on parameters of the eddy diffusivity, the most important challenge for usefulness to larger-scale modeling efforts.

Just to mention one example, in very many situations of interest, there is not only a background stratification, but also a large scale background (vertical) shear $S=\partial U / \partial x_{3}$, central to the turbulent production term $\mathcal{P}$. This background shear also defines a timescale, and thus a natural "background" parameter is an appropriate (gradient) Richardson number Ri, defined as

$$
\mathrm{Ri} \equiv \frac{N^{2}}{S^{2}} .
$$

In the ideal situation of steady, inviscid flow where the base (streamwise) flow only depends on $x_{3}$, the well-known Miles-Howard theorem [30,31] establishes that the necessary condition for the flow to be linearly unstable to normal mode disturbances is that somewhere within the flow $\operatorname{Ri}\left(x_{3}\right)<1 / 4$. Although only established in these highly restrictive circumstances, it seems plausible (and is often assumed) that "strong" stratification with large values of this parameter will tend to suppress the onset and maintenance of disordered motion. However, as discussed in more detail below, if there is significant spatiotemporal variation in $\mathrm{Ri}$, which inconveniently appears to be commonplace, then it can be very challenging to characterize a flow as being "strongly" stratified in any meaningful sense.

Interestingly, Ri emerges naturally even when considering an appropriate characterization of the mixing within a flow, specifically when considering the "turbulent" Prandtl number of a (vertically 
sheared) flow. Just as it is possible to define the eddy diffusivity of density $\kappa_{T}$ as in (5), the eddy diffusivity of momentum $v_{T}$ notionally relates the second order correlation of the Reynolds stress to the mean vertical shear (assumed to be positive and in the $x_{1}$ direction alone for simplicity) and so

$$
v_{T} \equiv \frac{-\left\langle u_{1}^{\prime} u_{3}^{\prime}\right\rangle}{\partial U / \partial x_{3}} \equiv \frac{\mathcal{P}}{S^{2}}
$$

on multiplying across above and below by the shear $S$ and remembering the definition of the turbulence production $\mathcal{P}$. Therefore, the turbulent Prandtl number $\operatorname{Pr}_{T}$ may be expressed as

$$
\operatorname{Pr}_{T} \equiv \frac{\nu_{T}}{\kappa_{T}}=\frac{\mathcal{P}}{\mathcal{B}} \frac{N^{2}}{S^{2}}=\frac{\mathrm{Ri}}{\mathrm{Ri}_{f}},
$$

using the definition (4) of the flux Richardson number.

Naturally, there are many ways to interpret this expression. A particularly helpful deduction can be drawn from consideration of a situation where $\mathrm{Ri}$ is sufficiently small so that the effect of the stratification may be considered "weak," in the specific sense that it is reasonable to assume (at least loosely) that the scalar is mixed similarly to the momentum, and so $\operatorname{Pr}_{T} \sim 1$. This implies inevitably that $\mathrm{Ri}_{f} \sim \mathrm{Ri}$, and so also $\mathrm{Ri}_{f}$ would be (in this circumstance at least) "small" and correlated to the characteristic value of $\mathrm{Ri}$.

Although appealing because of both its direct connection to the Miles-Howard theorem, and its relative practical accessibility to measurement in real flows, a possible criticism of the use of Ri as a way to parametrize turbulent mixing is that it is a ratio of timescales of the large-scale, background properties of the flow, with no apparent explicit information about the properties of the turbulence. However, as is discussed further below, there is mounting evidence that there are circumstances where the background shear and/or the stratification are effectively emergent quantities, inherently coupled to the turbulence, particularly when the flow is quasisteady in what Turner [32] referred to as "a kind of equilibrium." On the other hand, a reasonable argument can be presented [33] that a more natural parameter to consider is the (turbulent) Froude number, $\mathrm{Fr}_{T}$, effectively the ratio of characteristic timescales of the turbulence and the stratification, and defined as

$$
\operatorname{Fr}_{T} \equiv \frac{\mathcal{E}}{N \mathcal{K}^{\prime}}
$$

Therefore the fundamental parametrization requires (at least) the determination of the functional dependence of $\kappa_{T}\left(\operatorname{Pr}, \mathrm{Ri}, \mathrm{Fr}_{T}, \mathrm{Re}_{b}\right)$, although, as already noted many studies have focused on the (superficially at least) equivalent determination of the functional dependence on the same parameters of various definitions of the turbulent flux coefficient $\Gamma$.

Although in principle each of these parameters is independent as they consider different aspects of the fluid, the background, and the turbulent flow [34,35], it is important to be vigilant for the possibility of implicit correlations between the different variables, leading to certain areas of parameter space being possibly inaccessible. For example, if the stratification is "weak," while the turbulence is vigorous, then it seems reasonable that the dissipation rate will have the classical inertial scaling $\mathcal{E} \sim \mathcal{U}^{3} / \mathcal{L}$ with the turbulent kinetic energy $\mathcal{K}^{\prime} \sim \mathcal{U}^{2}$ for some characteristic velocity scale $\mathcal{U}$ and length scale $\mathcal{L}$. Therefore,

$$
\operatorname{Fr}_{T}^{-2} \equiv\left(\frac{N \mathcal{K}^{\prime}}{\mathcal{E}}\right)^{2} \sim \frac{N^{2}}{(\mathcal{U} / \mathcal{L})^{2}} \sim \frac{N^{2}}{S^{2}} \equiv \mathrm{Ri},
$$

under the further (at least plausible) assumption that the shear also scales with $\mathcal{U} / \mathcal{L}$. Furthermore, due to the reciprocal dependence on $N$, it is not immediately clear whether it is actually possible for a (sheared) turbulent flow to be simultaneously strongly stratified (with "large" $\mathrm{Ri}$ in some sense) and vigorously and intensely turbulent with "large" $\mathrm{Re}_{b}$, and indeed there is some evidence that $\mathrm{Ri}$ and $\mathrm{Re}_{b}$ can, at least in some circumstances, vary inversely relatively to each other [36]. It is clearly of great (and indeed pressing) interest to investigate this point further. 


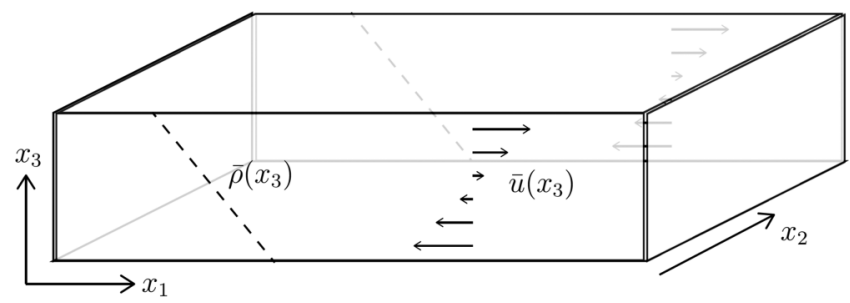

FIG. 1. Flow geometry considered in Ref. [29]. The horizontally averaged density and streamwise velocity are maintained as constant in time and with uniform gradient in the vertical direction, while the perturbations away from these averages are allowed to vary within a triply periodic box. The "strength" of the stratification is determined by the value of the coupling parameter $\mathrm{Ri}$ in (20), which is varied with time, effectively through allowing the strength of gravity to vary, until the turbulence settles down to a statistically steady behavior.

\section{FORCED (VERTICAL) SHEAR FLOWS: IS $\Gamma\left(\operatorname{Re}_{b}\right)$ ?}

Turning to perhaps the simplest possible dependence of the flux coefficient, it is (still) a controversial research issue whether an appropriate definition of the flux coefficient depends on $\mathrm{Re}_{b}$. In a deeply influential paper [9], Shih et al. [37] considered numerical simulations of homogeneous stratified sheared turbulent flows. The (mean) shear and buoyancy frequency were fixed, so that, for every simulation, Ri had a fixed constant value. However, not all the simulations were statistically steady. Data sampling a wide range of buoyancy Reynolds numbers were thus constructed through instantaneous spatial averaging. At the heart of such a process is the implicit assumption that data from such "snapshots" are still useful to identify parametric dependence of the various mixing properties. Using this approach, they obtained clear evidence of $\Gamma_{\mathcal{B}} \simeq 0.25$ (equivalent to $\mathrm{Ri}_{f} \simeq 0.2$, though the data scatter is definitely consistent with Osborn's $\Gamma_{\mathcal{B}} \sim 0.2$, in what they referred to as the "intermediate" range $7 \leqslant \operatorname{Re}_{b} \leqslant 100$. They also detected clear evidence of a scaling $\Gamma_{\mathcal{B}} \propto \operatorname{Re}_{b}^{-1 / 2}$ in what they referred to as the "energetic" regime of $\operatorname{Re}_{b} \gtrsim 100$. Such a decreasing scaling $\Gamma_{\mathcal{B}}\left(\operatorname{Re}_{b}\right)$ (or equivalently $\mathrm{Ri}_{f}$ ) at sufficiently large values of $\mathrm{Re}_{b}$ has also been identified in experimental and observational data [38].

However, typically other parameters did not remain constant, and spatial and temporal variability occurred naturally. Therefore, the possibility remained that the observed behavior at "high" $\operatorname{Re}_{b}$ was associated with correlated variation in other parameters, or alternatively temporal (or spatial) dependence. Also, as in passive scalar mixing [19], "history matters" in mixing, due to the subtle interplay between larger-scale "stirring" and irreversible, small-scale, and inherently diffusive "mixing." Inevitably, the previous stirring time-history leaves a long-lasting imprint on the subsequent mixing within a flow, and so using multiple "snapshots" to capture instantaneous mixing properties in a time-evolving flow must be treated with caution.

To isolate (as much as possible) the effect of variation in $\mathrm{Re}_{b}$ on mixing, Ref. [29] considered a very similar flow geometry to that considered in Ref. [37]. The computational domain had periodic boundaries in the horizontal. A constant density gradient and (streamwise) velocity shear are imposed in the vertical direction, with periodic perturbations in velocity and density away from these enforced background gradients imposed at the top and bottom of the computational domain. The flow geometry is shown in Fig. 1.

When appropriately nondimensionalized, the perturbation velocity and density satisfy the following equations:

$$
\begin{gathered}
\mathbf{u}_{\mathrm{tot}}=\overline{\mathbf{u}}+\mathbf{u}=x_{3} \hat{\mathbf{x}}_{1}+\mathbf{u} ; \quad \rho_{\mathrm{tot}}=\bar{\rho}+\rho=-x_{3}+\rho \\
\frac{\partial}{\partial t} \mathbf{u}+\mathbf{u} \cdot \nabla \mathbf{u}+u_{3} \hat{\mathbf{x}}_{1}+x_{3} \frac{\partial}{\partial x_{1}} \mathbf{u}=-\nabla p-\operatorname{Ri} \rho \hat{\mathbf{x}}_{3}+\frac{1}{\operatorname{Re}} \nabla^{2} \mathbf{u}, \\
\frac{\partial}{\partial t} \rho+\mathbf{u} \cdot \nabla \rho-u_{3}+x_{3} \frac{\partial}{\partial x_{1}} \rho=\frac{1}{\operatorname{RePr}} \nabla^{2} \rho
\end{gathered}
$$



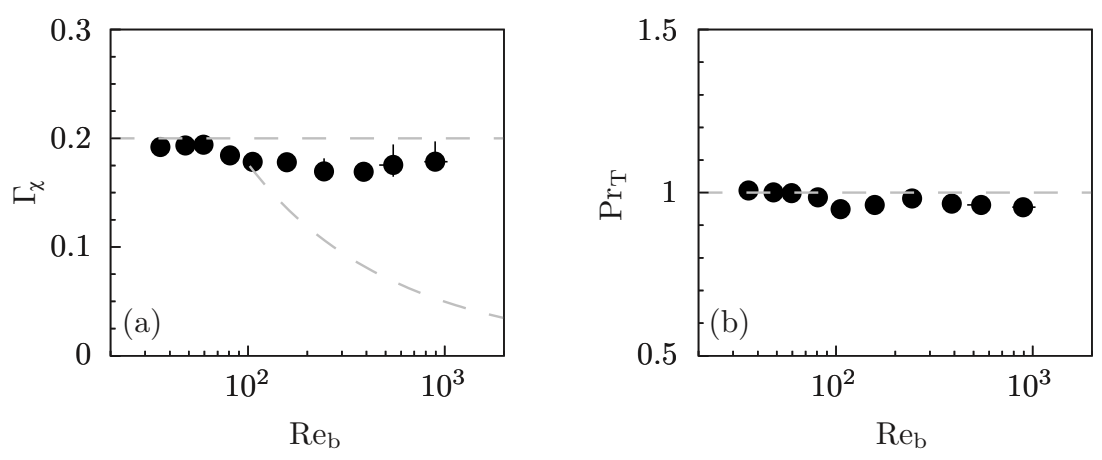

FIG. 2. Variation with $\operatorname{Re}_{b}$ of (a) $\Gamma_{\chi}$ and (b) $\operatorname{Pr}_{T}$ for statistically steady constant shear or constant density gradient simulations described in Ref. [29]. The $\mathrm{Re}_{b}^{-1 / 2}$ scaling proposed in Ref. [37] and the upper bound $\Gamma_{\chi}=0.2$ proposed in Ref. [10] are both shown with dashed lines in the left panel. Adapted from and used with permission from Ref. [29], copyright APS, all rights reserved.

When scaled in this fashion, the flow has three control parameters: $\operatorname{Pr}$ (always chosen to be $\operatorname{Pr}=1$ in these simulations), $\operatorname{Re}$ (which may be thought of as the nondimensional size of the computational domain or, equivalently, the inverse of the fluid's viscosity), and Ri. Crucially, Ri here is constant throughout the domain, with respect to both time and the vertical coordinate $x_{3}$, due to the imposed background velocity and density gradients. Ri essentially plays the role of a coupling parameter, quantifying the dynamical (buoyancy) effects of density perturbations on the velocity field.

Since the mean shear is imposed, such flows are continuously forced, with implicit power required to maintain this shear being balanced by both viscous dissipation and density flux through the system. A three-step iterative algorithm ensures that the flow evolved toward a statistically steady state. First, $\mathrm{Re}$ is chosen. Then a target turbulent kinetic energy $\mathcal{K}_{t}^{\prime}$ is chosen. Third, Ri is adjusted so that $\mathcal{K}^{\prime} \rightarrow \mathcal{K}_{t}^{\prime}$. If $\mathcal{K}^{\prime}$ increases, then $\mathrm{Ri}$ is increased to "damp down" the turbulence, while, conversely, if $\mathcal{K}^{\prime}$ decreases, then $\mathrm{Ri}$ is decreased to allow the turbulence to grow in a more weakly stratified environment. This process ensures that the flow is turbulent throughout the computational flow domain, and so there is also no ambiguity in the value of $\operatorname{Re}_{b}$ associated with the averaging of the dissipation rate chosen.

Provided the flow converges to a quasisteady state (which fortunately is observed to happen) varying $\mathcal{K}_{T}^{\prime}$ leads to several key quantities emerging naturally. First, the dissipation rate (and hence the value of $\mathrm{Re}_{b}$ ) emerges, and for the particular choices of $\mathrm{Re}$ and $\mathcal{K}_{t}^{\prime}$ varies in the range $36 \leqslant \mathrm{Re}_{b} \leqslant 900$. More interestingly, the particular critical value $\mathrm{Ri}_{c}$ at which steady state occurs nevertheless varies over a very narrow range $0.146 \leqslant \mathrm{Ri}_{c} \leqslant 0.163$, as indeed does the analogously emergent value of the turbulent Froude number $0.42 \leqslant \operatorname{Fr}_{T} \leqslant 0.52$. Therefore, to a good approximation, these statistically steady simulations (perhaps fortuitously) access a very wide range of $\operatorname{Re}_{b}$, while to a good approximation keeping all the other relevant parameters constant.

Furthermore, due to the statistical steadiness, both $\chi \simeq \mathcal{B}$, and perhaps more importantly, the partitioning between $\mathcal{E}$ and $\mathcal{B}$ (or equivalently $\chi$ ) emerges without being set a priori. In other words, the "taxation rate" of the stratification quantified by the turbulent flux coefficient $\Gamma_{\chi}$ can be calculated. The results are plotted in Fig. 2. Although there is a slight decrease at higher $\operatorname{Re}_{b} \gtrsim 300$, there is no evidence of the $\mathrm{Re}_{b}^{-1 / 2}$ scaling, and the data are really very close to the Osborn upper bound [10]. Furthermore, as shown in the right panel, $\operatorname{Pr}_{T} \simeq 1$ across the full range of $\operatorname{Re}_{b}$. An (admittedly highly speculative) interpretation of these data is that statistically steady (vertically) shear-driven turbulence in a stratified fluid can be supported at the highest possible value of $\mathrm{Ri}$ at which $\operatorname{Pr}_{T} \simeq 1$, thus tending toward a situation where $\mathrm{Ri} \sim \operatorname{Ri}_{f} \sim 0.17$ and hence $\Gamma \simeq 0.2$, precisely as conjectured by Osborn [10]. Of course, $\operatorname{Pr}_{T} \simeq 1$ can reasonably be characterized as a "weakly stratified" flow. 
The flow geometry shown in Fig. 1 is highly idealized. Perhaps the most serious criticism is that the imposed forcing is both quite artificial, and imposes a tendency toward a single characteristic value of $\mathrm{Ri}$, thus making it impossible to determine whether the observation that $\operatorname{Pr}_{T} \simeq 1$ is the expected generic property of sufficiently weakly stratified flows (and hence that the original inequality $\Gamma \leqslant 0.2$ is actually appropriate for statistically steady flows [10]). Furthermore, only a single value of the (molecular) Prandtl number $\operatorname{Pr}=1$ is considered, and it is at least conceivable that varying $\operatorname{Pr}$ may lead to variation in $\operatorname{Pr}_{T}$, at least in some circumstances.

Fortunately, many of these criticisms can be addressed by considering (vertically) stratified plane Couette flow. This is the flow between two horizontal bounding plates, separated by a distance $2 h_{0}$, forced to move at equal and opposite speeds $\pm U_{0}$ (thus defining a Reynolds number $\operatorname{Re}_{0}=U_{0} h_{0} / v$ ) and (notionally) maintained at different temperatures (with the hotter plate at the top) so that Dirichlet boundary conditions are imposed on the velocity and density $\left(\rho_{r} \pm \rho_{0}\right)$ of the fluid, thus also defining a "bulk" Richardson number $\mathrm{Ri}_{b}=g \rho_{0} h_{0} /\left(\rho_{r} U_{0}^{2}\right)$. Such a flow has several computational attractions, not least of which is that there is naturally a constant (with height) vertical density flux [39]. The value of this flux naturally emerges from the near-wall structure of the density field, just as the wall shear stress (and hence the power injection by the wall-forcing) naturally emerges from the near-wall structure of the horizontally averaged velocity field.

These properties can be exploited to construct a Monin-Obukhov (M-O) similarity theory for the mean velocity and density profiles [40,41], which can be further generalized to consider fluids with different molecular Prandtl numbers in the range $0.7<\operatorname{Pr}<70$ [39]. This similarity theory proves to be highly appropriate to a description of the calculated mean properties of the numerical simulations, and in particular coupled, essentially linear profiles in density and velocity emerge (outside of thin boundary layers). These linear profiles imply that, throughout the interior of the flow, specific values of the (gradient) Richardson number Ri emerge with little vertical variation.

The theory predicts (and the simulations confirm) that this interior $\mathrm{Ri}$ is bounded above by $\mathrm{Ri}_{c} \simeq$ 0.21 , effectively irrespective of the value of $\mathrm{Re}_{0}$, and that the flow becomes intermittent (in a wellpredicted way) if the flow becomes too strongly stratified in the sense that the "Obukhov length" (the ratio of the cube of the friction velocity to the density flux) becomes too small, as predicted in Ref. [42]. However, for the region of parameter space where the flow remains turbulent, irrespective of the value of the molecular $\operatorname{Pr}_{1} \operatorname{Pr}_{T} \simeq 1$ over the entire range of $\mathrm{Ri} \lesssim \mathrm{Ri}_{c}$, and so $\mathrm{Ri}_{f} \simeq \mathrm{Ri} \leqslant$ $\mathrm{Ri}_{c} \simeq 0.21$, once again highly consistently with Ref. [10].

As pointed out in Ref. [41], the M-O theory also predicts the value of $\mathrm{Re}_{b}$, thus casting into serious doubt whether it is appropriate (at least in this flow geometry) to consider $\operatorname{Re}_{b}$ to be the key independent parameter to describe the mixing properties. Crucially, even for this much more realistically forced flow, the stratification can always be characterized as being "weak" in that $\operatorname{Pr}_{T} \simeq$ 1. Further evidence of this weakness is demonstrated by the (empirical) fact that, as described above, the interior (emergent) $\mathrm{Ri} \propto \mathrm{Fr}_{T}^{-2}$, consistent with the stratification not (significantly) modifying the classical inertial scaling for the turbulent dissipation rate $\mathcal{E}$. However, it remains somewhat mysterious that the effect of the stratification might be considered to be weak, until that is, it just stops being weak, and causes the flow to become intermittent, although it is the specific properties of the flow near the wall which appear to be key to the onset of intermittency.

Furthermore, this flow highlights a common challenge in consideration of stratified shear flows, namely ambiguity and the associated potential for confusion in the particular choice of Richardson number to describe the flow. The interior gradient Richardson number Ri emerges as a consequence of the balances at the heart of the M-O theory, and in general this is distinct from the bulk Richardson number $\mathrm{Ri}_{b}$ set by the boundary conditions. Finally, it is still mysterious why $\mathrm{Ri}_{c} \simeq 0.2$, with, as noted in Ref. [39], the relationship to the Miles-Howard theorem being "apparently fortuitous."

\section{UNSTABLE (VERTICAL) SHEAR FLOWS: DOES HISTORY MATTER?}

The forced flows considered by both Ref. [29] and Ref. [39] were constructed (and in particular forced) deliberately in an attempt to observe statistically steady flows so that the mixing properties 
could be isolated from any transient (and hence inherently time-dependent) effects. Also, both flows allowed for characteristic values of the Richardson number to be identified unambiguously. Of course, transient flow evolution is exceptionally common. Furthermore, the whole paradigm of mixing events which go through several stages starting with flow instability leading to turbulence transition, vigorous mixing and turbulence with final ultimate decay, is based on the sequential occurence of highly time-dependent phenomena.

Indeed, it has been commonly assumed that a generic way in which turbulent stratified mixing occurs is through the onset and subsequent breakdown of shear instabilities in a stratified flow. This view naturally leads to the constraints implied by the Miles-Howard theorem, and so the consideration of flows which (at least somewhere) have local gradient Richardson number Ri significantly less than $1 / 4$, although there are some important subtleties associated with "optimal" transient growth [43,44], ambient turbulence [45], finite amplitude perturbations [46,47], and finite Reynolds-number effects [48]. Indeed, much of the attention has been focused on stratified flows with inflectional velocity profiles which would be unstable even in the absence of stratification, even though, due not least to the presence of internal waves, statically stable stratification can lead to instability in flows which are linearly stable in the absence of stratification (see, for example, Ref. [49]).

A particularly instructive class of flows which has been widely considered $[16,17,50,51]$ have initial hyperbolic tangent profiles in streamwise velocity and density:

$$
\mathbf{u}_{b}=U\left(x_{3}\right) \hat{\mathbf{x}}_{1} ; \quad U\left(x_{3}\right)=U_{0} \tanh \left(x_{3} / d_{0}\right) ; \quad \rho_{b}=\rho_{r}-\rho_{0} \tanh \left(x_{3} / \delta_{0}\right) ; \quad \operatorname{Re}_{0}=\frac{U_{0} d_{0}}{v} .
$$

Here $2 d_{0}$ is the initial total depth of the shear layer (which might more consistently be called a vorticity interface) while $2 \delta_{0}$ is the initial total depth of the density interface, and $\rho_{r}$ is a reference density. There is a total velocity jump of $2 U_{0}$, leading to a natural definition of the flow Reynolds number as $\operatorname{Re}_{0}=U_{0} d_{0} / \nu$. Care must be taken when making comparisons, as some studies use the total shear layer depth and velocity jump, thus leading to a Reynolds number larger by a factor of four.

Consideration of these flows requires the analysis of an initial value problem. Whereas the flows considered in Sec. III are continuously forced with a forcing which is either constant, or at least very close to constant with time, the evolution of these flows evolves "freely" from an artificially chosen and inherently unstable initial condition. In a sense, this initial condition has been set up by some forcing, which is "switched off" at $t=0$ when the flow (with some small perturbation) is allowed to evolve. These two different situations constitute two end members of the more general (and certainly more geophysically relevant) class of flows with "forcing" which can vary in both space and time. It is always important to remember just how idealized such initial value problems actually are, and whether their evolution leads to generic behavior is an interesting, and still open question. This issue is discussed further in Sec. VI.

A further potential source of confusion is the significance of the Richardson number of such flows. The background gradient Richardson number is

$$
\operatorname{Ri}\left(x_{3}\right) \equiv \frac{N^{2}}{S^{2}}=\frac{g \rho_{0} d_{0}}{\rho_{r} U_{0}^{2}} \frac{d_{0}}{\delta_{0}} \frac{\operatorname{sech}^{2}\left(x_{3} / \delta_{0}\right)}{\operatorname{sech}^{4}\left(x_{3} / d_{0}\right)} \equiv \operatorname{Ri}_{b} R \frac{\operatorname{sech}^{2}\left(x_{3} / \delta_{0}\right)}{\operatorname{sech}^{4}\left(x_{3} / d_{0}\right)},
$$

defining the length-scale ratio $R$ and the bulk Richardson number $\mathrm{Ri}_{b}$. Only for the simplest case of $R=1$ does $\mathrm{Ri}_{b}$ correspond also to the minimum gradient $\mathrm{Richardson}$ number $\mathrm{Ri}_{m}$ (at the midpoint of the shear layer), the relevant quantity for the application of the Miles-Howard theorem.

The particular value of the length scale ratio $R$ plays a key role [52] in the relevance of $\mathrm{Ri}_{b}$ to the value of $\mathrm{Ri}_{m}$. In particular, if $R$ is large, and so the density interface can be characterized as "sharp," Ri drops to small values away from the interface, and so $\mathrm{Ri}_{b}$ (and indeed the Miles-Howard theorem) becomes irrelevant to determining whether the flow is unstable or not. Large values of $\mathrm{Ri}_{b}$ may suggest that the flow is "strongly" stratified, but as there are regions of the flow where Ri is very small, there is no obvious blanket constraint against the flow being unstable. 
In stratified shear flows, this is not just a point of academic curiosity. Although hyperbolic tangent profiles were initially considered due to the fact that significant analytical progress could be made [30], they are also close to the error function profiles which would be expected to develop due to diffusion between two streams of fluid with different properties of velocity and density. In such a circumstance, the ratio $R \propto \sqrt{\operatorname{Pr}}$ [52], and so it is reasonable to assume that it is at least possible for relatively "sharp" interfaces to occur in water for example [with $\operatorname{Pr} \sim O(10)$ ] while $R \sim O(1)$ is expected in gases $(\mathrm{Pr} \simeq 0.7$ in air).

\section{A. Overturning and scouring: KHI and HWI}

These two different possible situations suggest two qualitatively different types of mixing, which may be referred to as "overturning" or "scouring" [53]. If the stratification is relatively weak, then a shear instability (and its ensuing turbulent breakdown) can overturn the density interface, further smoothing and spreading out the density distribution. In such a case, it seems at least plausible that the ensuing mixing could appropriately be modelled as a diffusive process, due to the fact that it (further) smears out the density gradient in the vicinity of the interface. On the other hand, if the density interface is sufficiently "strong" and "sharp," then instabilities and turbulence are no longer sufficiently energetic to overturn the interface but rather are characterized by vortical structures that impinge on the interface and scour relatively fine wisps of fluid from the interface into the interior. This process could reasonably (if loosely) be characterized as "antidiffusive," as the interface not only can survive but may indeed be sharpened further in certain circumstances.

Although this is argument seems highly speculative, it is possible to identify circumstances under which such "scouring" dynamics might be expected to occur in a fully turbulent flow [54], and provided the flow actually starts in such a "layer-interface" state (also sometimes referred to as a "staircase"), such an interface can continue to persist in stratified plane Couette flow [55]. Furthermore, there also are primary instabilities of a stratified flow which exhibit these contrasting properties, establishing that this dichotomy is a useful way of thinking about stratified shear flows, even from an initial value viewpoint. Sufficiently weakly stratified flows are prone to the classical "overturning" Kelvin-Helmholtz instability (KHI), which at finite amplitude takes the form of a periodic array of elliptical vortices prone (at sufficiently high $\operatorname{Re}_{0}$ ) to a "zoo" of secondary instabilities [56,57]. These secondary instabilities rapidly trigger the transition to an intense burst of turbulence which "flares" for a relatively short period before decaying.

Conversely, generically when the scale ratio $R$ is sufficiently large so that $\mathrm{Ri}$ drops to small values away from the density interface, when the interface is sufficiently strong so that $\mathrm{Ri}_{b}$ is still sufficiently large, a qualitatively different "scouring" instability occurs. This instability is often referred to as the Holmboe wave instability (HWI) [58], though there is not the same complete consensus about nomenclature [59], and once again, the subtle effects of viscosity must be treated with care when classifying stratified shear instabilities [48]. In the simplest symmetric case when the density interface is at the midpoint of the shear layer, at finite amplitude the saturated primary HWI manifests as counterpropagating trains of patches of vorticity above and below the interface. These trains perturb the interface to form characteristic counterpropagating cusped waves, "scouring" wisps of fluid upward and downward from the cusps of these waves [60]. (The situation is somewhat more complicated when the symmetry is broken by the interface being displaced from the midpoint of the shear layer [61], with different dominant wavelengths and growth rates for the two branches of HWI propagating in different directions.)

\section{Mixing properties of "scouring" turbulence induced by HWI: Do flows self-organize?}

Although there has not been the same detailed analysis of the secondary instabilities of HWI, provided they are conducted at sufficiently high Reynolds number, numerical simulations [28,62] suggest that HWI are also prone to a wide range of secondary instabilities, and also breakdown to disordered turbulence. However, it appears that this turbulent phase "burns" for a significantly longer period of time compared to flows primarily prone to KHI. Indeed, for a significant period of 

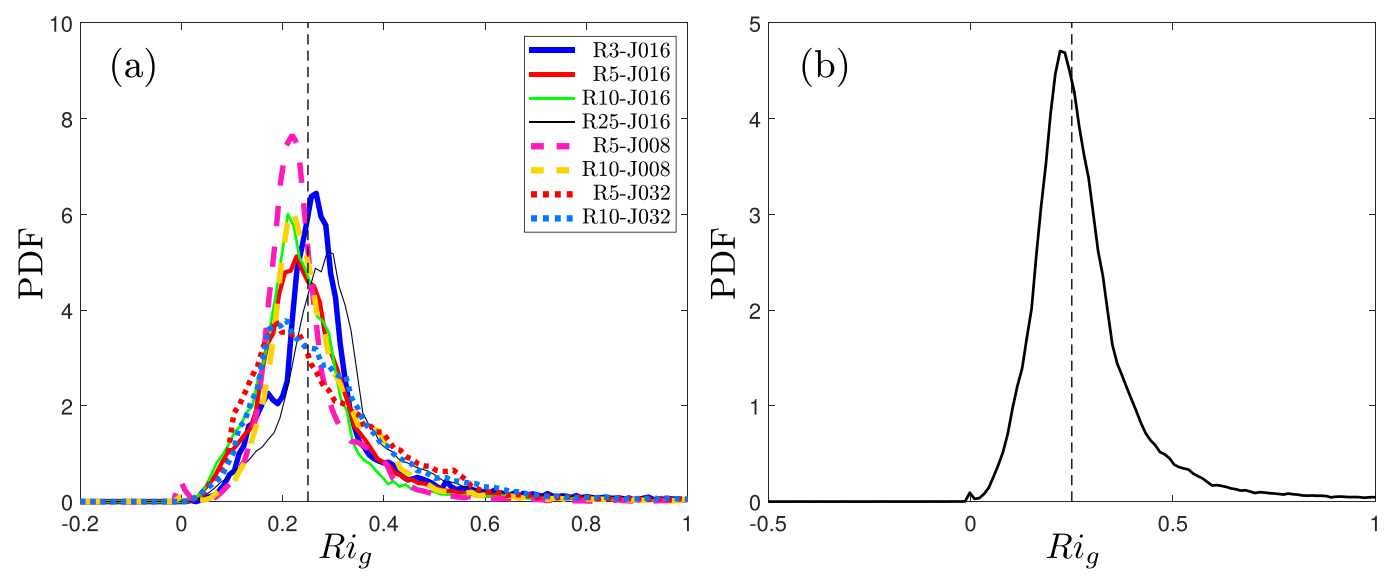

FIG. 3. (a) The probability density function (PDF) of $\mathrm{Ri}_{g}\left(x_{3}, t\right)$ for times during the turbulent period of eight different simulations prone to primary HWI with $\mathrm{Re}_{0}=6000$ and $\operatorname{Pr}=8$. Each line type corresponds to a different initial condition choice of length-scale ratio $R$ and bulk Richardson number $\mathrm{Ri}_{b}$ (coded as $\mathrm{J}$ ). (b) The aggregated probability density function of $\mathrm{Ri}_{g}\left(x_{3}, t\right)$ for times during the turbulent period of all eight different simulations. The vertical dashed line marks the critical value of 0.25 . Used with permission from Ref. [62], copyright CUP, all rights reserved.

time, the turbulence induced by the HWI appears to relax into "a kind of equilibrium" as postulated by Turner [32], and this equilibrium turbulent state appears to be generic, in the sense that certain key characteristics of this turbulent state do not appear to depend on either the initial sharpness (quantified by the ratio $R$ ) or strength (quantified by the bulk Richardson number $\mathrm{Ri}_{b}$ ) of the density interface.

One way to consider quantitatively the generic character of the turbulence following the breakdown of primary HWI is to calculate the notional gradient Richardson number distribution $\mathrm{Ri}_{g}\left(x_{3}, t\right)$ associated with horizontally averaged profiles of streamwise velocity and density [62]. At every instant after the onset of the fully turbulent state, it is possible to calculate (from the data of a numerical simulation) the horizontally averaged density distribution $\bar{\rho}\left(x_{3}, t\right)$ and streamwise velocity $\bar{u}\left(x_{3}, t\right)$, and hence $\operatorname{Ri}_{g}\left(x_{3}, t\right)$ :

$$
\operatorname{Ri}_{g}\left(x_{3}, t\right) \equiv \frac{-\frac{g}{\rho_{r}} \frac{\partial \bar{\rho}}{\partial x_{3}}}{\left(\frac{\partial \bar{u}}{\partial x_{3}}\right)^{2}},
$$

where as usual $\rho_{r}$ is the reference density.

It is then possible to construct the probability density function (PDF, sampled at vertical locations and time) of this notional Richardson number distribution during the turbulent period of simulations initialized with a range of choices of $R$ and $\mathrm{Ri}_{b}$. The PDFs for eight different simulations (with different choices of $R$ and $\mathrm{Ri}_{b}$ ) are plotted independently in Fig. 3(a) and combined together in Fig. 3(b) [62]. Irrespective of the initial conditions chosen, the PDF for flows with HWI are very strongly peaked around the critical value of $\mathrm{Ri}_{g}=0.25$, marked in each panel with a vertical dashed line. Although not shown, equivalent PDFs for simulations prone to primary KHI do not exhibit such universal, "critical" behavior. This behavior of HWI-unstable flows is highly suggestive of the flow self-organizing critically into a marginally unstable state for a significant time. Indeed, other aspects of the flow (such as apparent scale invariance in the "avalanching" eddies) are also suggestive of such flows being attracted toward a generic state of self-organized criticality.

These key aspects of universal, long-lived turbulence properties also appears to lead to mixing properties which have some universality. Within such flows, as there still is undoubtedly a life cycle to the mixing event, it is most natural to consider cumulative measures of mixing, as defined in (7). 


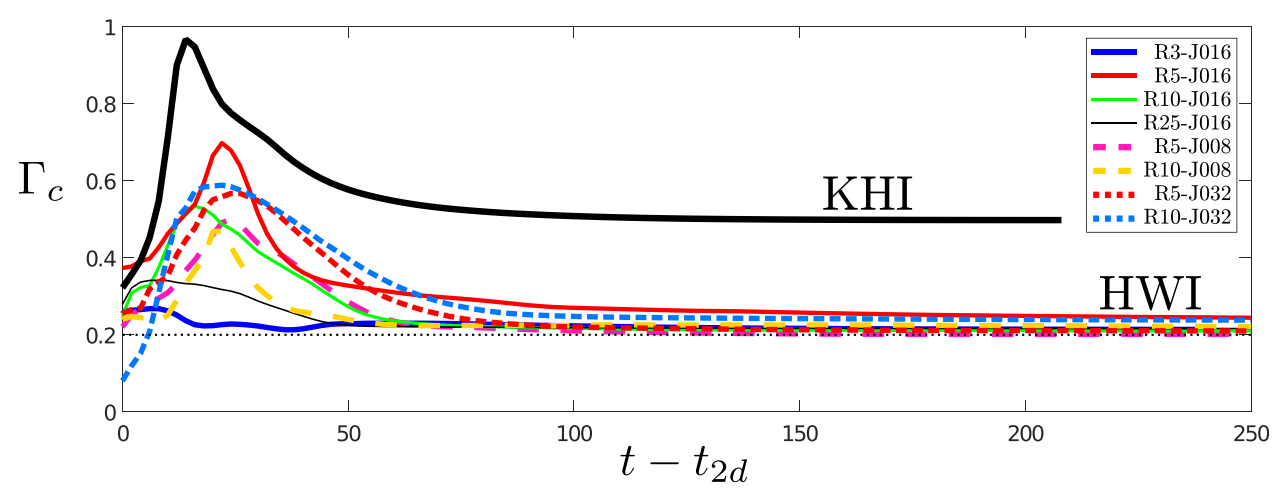

FIG. 4. Time variation of cumulative turbulent flux coefficient $\Gamma_{c}(t)$ as defined in (7) for the eight simulations listed in Fig. 3 prone to primary HWI (plotted with the same line types) and for a simulation with $\mathrm{Re}_{0}=6000, \mathrm{Pr}=8, \mathrm{Ri}_{b}=0.16$, and $R=1$ prone to primary KHI (plotted with a solid black line). Adapted from and used with permission from Ref. [62], copyright CUP, all rights reserved.

Therefore, in Fig. 4, $\Gamma_{c}(t)$ is plotted with different colored lines for these eight simulations, where $t_{0}=t_{2 d}$, i.e., the saturation time of the primary HWI. Interestingly, yet again the mixing within these flows converges (strongly) toward the upper bound proposed by Osborn [10] $\Gamma_{c} \rightarrow 0.2$. This is consistent again with $\operatorname{Pr}_{T} \sim O(1)$, and that such mixing may be thought of as being in some sense a quasisteady state for still "weak" stratification, when the most appropriate definition of Richardson number is used, and there is at least suggestive observational evidence consistent with this self-organized criticality argument [63].

\section{Mixing properties of "overturning” turbulence induced by KHI: How large can $\Gamma$ be?}

However, as is apparent in Fig. 3, the mixing properties of flows prone to primary KHI appear to be markedly different. The equivalent time evolution of $\Gamma_{c}$ is plotted with a black line for a flow with $\mathrm{Ri}_{b}=0.16$ and $R=1$ after the saturation of the primary Kelvin-Helmholtz billow. There are two key differences. First, $\Gamma_{c}$ "flares" to a much larger peak value than for any of the HWI-unstable flows. This flare is associated with the effects of the breakdown of the large primary overturning, and is perhaps not surprising, as such "convective" and statically unstable mixing processes are well known to be significantly more efficient than shear-driven mixing events [12]. Second, this large $O(1)$ peak value has a lasting imprint on $\Gamma_{c}$, with $\Gamma_{c} \sim 0.4$ for this flow for large times as the mixing event dies away, suggesting that mixing induced by the breakdown of the primary overturning of KHI may be characterized as being "optimal" [51].

Clearly, for such large overturning-driven mixing events, the flow history really matters, and the mixing can be much more efficient than postulated in Ref. [10]. As discussed in detail in Ref. [50], these discrepancies are not entirely surprising, since the underlying assumptions of the existence of a statistically steady homogeneous turbulent state with no coherent large scales or other significant advective terms are essentially all violated by the relatively rapid turbulent breakdown of KelvinHelmholtz billows. Furthermore, since "history does matter" for such mixing events, great care must be taken in interpreting data constructed from snapshots at different stages in the flow evolution.

Nevertheless, although the underlying assumptions of Ref. [10] do not necessarily apply, there is at least suggestive evidence that mixing associated with such shear-driven overturnings is relevant to real flows. Using "snapshots" from numerical simulations (with associated instantaneous values of mixing properties and parameters, such as $\mathrm{Re}_{b}$ ) it is possible to identify a certain characteristic dependence of turbulent flux coefficient $\Gamma_{\mathcal{M}}$ as a function of $\operatorname{Re}_{b}$, as shown in Fig. 5(a). The variation is clearly nonmonotonic, and fits well in an envelope defined by two expressions of the 

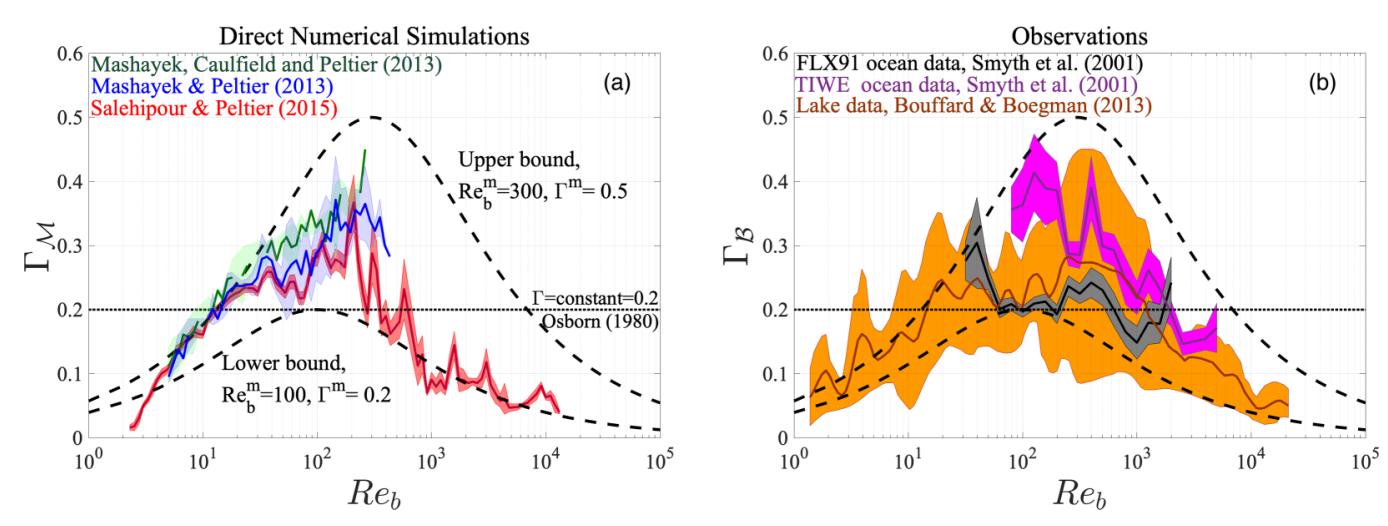

FIG. 5. Variation with $\mathrm{Re}_{b}$ of turbulent flux coefficients: (a) $\Gamma_{\mathcal{M}}$ inferred from numerical simulations $[16,50,64]$; (b) $\Gamma_{\mathcal{B}}$ inferred from observations $[65,66]$ as labeled. Shading quantifies estimates of uncertainty: for further details see Ref. [67]. The dashed lines show bounding expressions of the form (25) with $\operatorname{Re}_{b}^{m}=300, \Gamma^{m}=0.5$ and $\operatorname{Re}_{b}^{m}=100, \Gamma^{m}=0.2$. The horizontal dotted line marks the upper bound $\Gamma=0.2$ postulated in Ref. [10]. Adapted from and used with permission from Ref. [67], copyright Wiley, all rights reserved.

form

$$
\Gamma_{\mathcal{M}}\left(\operatorname{Re}_{b}\right)=\frac{2 \Gamma^{m}\left(\frac{\operatorname{Re}_{b}}{\operatorname{Re}_{b}^{m}}\right)^{1 / 2}}{1+\left(\frac{\operatorname{Re}_{b}}{\operatorname{Re}_{b}^{m}}\right)},
$$

where $\operatorname{Re}_{b}^{m} \sim O(100)$ is the value of $\operatorname{Re}_{b}$ at which $\Gamma_{\mathcal{M}}\left(\operatorname{Re}_{b}\right)$ attains its maximum value $\Gamma^{m}$. The upper bound has $\operatorname{Re}_{b}^{m}=300$ and $\Gamma^{m}=0.5$, while the lower bound has $\operatorname{Re}_{b}^{m}=100$ and $\Gamma^{m}=0.2$. Note that this functional form is consistent with the large $\operatorname{Re}_{b}^{-1 / 2}$ scaling [37,38], and also importantly consistent with oceanic and lake observations of $\Gamma_{\mathcal{B}}$ as shown in Fig. 5(b). (Shading typically denotes data scatter, as described in more detail in Ref. [67].) Essentially, such inherently transient mixing associated with overturning can be "efficient" (perhaps even "optimal" [51]) in that flux coefficients can be significantly larger than 0.2 , and also flux coefficients can decrease as $\operatorname{Re}_{b}$ becomes very large. The high values of flux coefficient are caused by the effects of the large overturning induced by the primary instabilities, demonstrating that such flows are profoundly affected by their time history. Also, and crucially, their behavior is still fundamentally associated with "weak" stratification, as the requirement for the flow to become unstable to KHI is inevitably constrained by the Miles-Howard theorem.

\section{V. "STRONGLY" STRATIFIED FLOWS: WHAT HAPPENS AS Fr $\mathrm{F}_{T} \rightarrow 0$ ?}

There are at least two "obvious" ways by which it is possible to consider flows which at least have the potential to be strongly stratified: flows where the background velocity gradients and density gradients are not parallel and numerical simulations where energy is injected at relatively large scales into a turbulent stratified fluid with arbitrarily strong stratification in an artificial, yet still controlled, way.

\section{A. Horizontal shear flows: Is layering generic?}

Flows where the velocity shear is horizontal and hence perpendicular to the (background) density gradient have a particular attraction for accessing turbulence in "strongly" stratified fluid. Clearly, the evolution within the flow of small perturbations is not constrained by the Miles-Howard theorem. Furthermore, the possible "roll-up" of the background shear into relatively isolated vortices does not 
have an inevitable energetic cost as in vertically sheared flows. Therefore, in principle at least, it is possible for horizontally sheared flows to be unstable for arbitrarily strong (vertical) stratification, although it is now known that the vertical stratification still has a strong effect on the instability properties $[68,69]$.

Indeed, even when vertically oriented vortices manage to develop, such vortices are prone to the "zig-zag" instability [70-72]. A key property of this instability is that it has a characteristic vertical length scale of $U / N$, where $U$ is some characteristic velocity of the flow. Subsequent breakdown at finite amplitude, inevitably (at sufficiently high Reynolds number) inducing turbulent mixing leads to a layer-interface structure, with relatively deep, relatively well-mixed "layers" (of scale $U / N$ ) separated by thinner "interfaces" of locally enhanced density gradient $[36,73,74]$.

Such a layer-interface structure occurs very commonly in stratified turbulent flows, with a particular dramatic manifestation occuring in stratified Taylor-Couette flow [75]. Initially vertically linearly stratified fluid in the annular region between two concentric cylinders spontaneously forms such a layer-interface structure when the inner cylinder is rotated at sufficiently high rotation rate relative to the outer (stationary) cylinder. Evidence suggests that the initial depth of the layers in this "staircase" is set by the onset of the "stratorotational" instability [76,77], but the subsequent flow can be very strongly turbulent, with vigorous turbulence with the relatively well-mixed layers scouring (and potentially intermittently overturning [78]) the much more strongly stratified "interfaces."

It can be tempting to classify such layered flows as being strongly stratified, in that estimates of the turbulent Froude number $\operatorname{Fr}_{T} \ll 1$, when based on volume averages of dissipation rate $\mathcal{E}$ in the numerator and turbulent kinetic energy $\mathcal{K}^{\prime}$ and buoyancy frequency $N$ in the denominator. However, using such uniform values for a highly spatially variable flow can be misleading, especially if used as evidence that vigorous turbulence can be maintained in such strong stratification, and can lead to nontrivial irreversible mixing. In particular, the experimental evidence $[75,78,79]$ that the vertical density flux (and hence appropriate measures of mixing efficiency) does not depend on the strength of (very strong) stratification (as quantified by overall estimates of $\mathrm{Fr}_{T}$ ) should not be interpreted as convincing evidence that nontrivial mixing is maintained as $\mathrm{Fr}_{T} \rightarrow 0$. Vigorous turbulence is generically localized in the relatively well-mixed (and hence relatively weakly stratified) layers, rather than over the (arbitrarily strongly stratified) "sharp" interfaces.

Unfortunately, stratified Taylor-Couette flow does not establish categorically whether turbulence can still remain in the "strongly stratified turbulence" regime (sometimes also called the "layered anisotropic stratified turbulence" or LAST regime [80]). This regime is closely associated with a self-similar scaling [81] that naturally leads to layers of depth $U / N \equiv L_{v}$, and is characterized by a particular distinguished limit. This limit requires that both the (horizontal) Reynolds number $\operatorname{Re} \equiv U L_{h} / v \gg 1$ and the horizontal Froude number $F_{h} \equiv U /\left(L_{h} N\right) \ll 1$ (where $U$ is a characteristic velocity scale and $L_{h}$ is a characteristic horizontal scale) such that $\operatorname{Re} F_{h}^{2} \gg 1$. With the further assumption that $\mathcal{E} \sim U^{3} / L_{h}$ (i.e., that the dissipation has the classical inertial scaling, which could perhaps be criticized in a flow with "strong" stratification) this distinguished limit implies (among other characteristics [82]) that $\operatorname{Re}_{b} \gg 1$, As already noted, $\operatorname{Re}_{b} \gg 1$ is associated with a high dynamic range with large-scale separation between the Kolmogorov microscale $L_{K}$ and the Ozmidov length scale $L_{O}$ as defined in (13). Using the inertial scaling and the scaling that $F_{h} \ll 1$, the characteristic scales are thus naturally ordered as $L_{h} \gg L_{v} \gg L_{o} \gg L_{K}$.

There are reasonable arguments that this regime is of great geophysical significance, but unfortunately it is extremely computationally demanding to simulate [83,84]. Furthermore, due not least to the predicted inherently anisotropic structure of high-aspect ratio "pancakes" of turbulence (as $L_{h} \gg L_{v}$ ), there is a clear need to identify the turbulence and mixing properties of different regions within such inherently anisotropic, spatiotemporally variable flows. This identification would be a natural application for a data-driven approach that could potentially be "learnt" using modern artificial intelligence algorithms [85]. 


\section{B. Forced "strongly stratified" flows: Are internal waves efficient mixers?}

An alternative approach attempting to access strongly stratified flow regimes, in particular avoiding the need to compute in horizontally extended computational domains, is to consider computations with artificial body-forcing at large scales. Other attractions of this approach are that it allows a rational sweeping through parameter space in a controlled fashion varying the turbulent Froude number $\operatorname{Fr}_{T}$ without a reliance on shear-driven instabilities, and also it yields at least the possibility that the flow can approach a statistically steady state. This latter aspect removes (presumably) the possibility that the time-history of the flow will matter in determining the turbulence (and mixing) properties of the flow. Once again, it is important to remember that this form of forcing is (at least quasi) steady in time. Spatiotemporal variability does indeed emerge, but it is not possible to gain insight into the dynamics of flows with (surely more realistic) spatiotemporally variable forcing.

In an important paper [33], this quasisteadily forced approach was used to investigate the (hypothesized) dependence $\Gamma_{\mathcal{B}}\left(\mathrm{Fr}_{T}\right)$. For large $\mathrm{Fr}_{T}$, the scaling $\Gamma_{\mathcal{B}} \propto \mathrm{Fr}_{T}^{-2}$ is clearly identified, entirely consistently with the weak stratification scaling $\mathrm{Ri}_{f} \propto \mathrm{Ri}$ discussed above, associated with the properties of the turbulence indeed being largely unaffected by the stratification, and also consistent with previous experimental and observational studies, as compiled in, for example, Ref. [86]. At intermediate $\mathrm{Fr}_{T} \sim O(1), \Gamma_{\mathcal{B}} \simeq 0.5$ a maximum value in this study [33], before apparently reducing to smaller, yet categorically positive, and essentially constant independent of $\operatorname{Fr}_{T}, \Gamma_{\mathcal{B}} \simeq 1 / 3$ as $\mathrm{Fr}_{T} \rightarrow 0$. Reassuringly, the high $\mathrm{Fr}_{T}$ scaling and the existence of a constant $\mathrm{Fr}_{T}^{0}$ scaling as $\mathrm{Fr}_{T} \rightarrow 0$ has been subsequently confirmed to apply for a wide range of different flows [87]. However, when data are collated from different flows, there is no clear evidence of nonmonotonicity at intermediate $\mathrm{Fr}_{T}$, and also the small $\mathrm{Fr}_{T}$ asymptote is consistent with $\Gamma \sim 0.5$, reminiscent of the peak value previously observed [33] and also in flows prone to primary KHI [67].

This is at least slightly suggestive of two conjectures. First, it suggests that the particular form of forcing may indeed be significant, at least for the particular numerical value of flux coefficient associated with the induced turbulence. Second, it suggests that the "taxation rate" of one-third of injected large-scale energy eventually leading to irreversible mixing and two-thirds being dissipated viscously may be accessible for such (at least apparently) strongly stratified flows. Of course, to investigate the significance of these conjectures, it is natural to consider the mixing properties of stratified turbulent flows forced (at large scales) using a variety of different strategies.

It is (perhaps unsurprisingly) straightforward to establish that the particular numerical value of the (nonzero) asymptotic value of an appropriately defined $\Gamma$ as $\mathrm{Fr}_{T} \rightarrow 0$ does indeed depend on the forcing strategy utilized [88]. However, it is also important to consider (admittedly artificial) bodyforcing which has at least some potential basis in reality. One (reasonable) choice injects energy at large scale into purely horizontal (and hence in some sense "vortical") modes [89]. An alternative attractive choice is to model the large-scale forcing as being associated with the internal wave field. Such a forcing can be designed both to have a spectral wave-number dependence designed to mimic that which is observed geophysically and also for the different velocity and density components to be inter-related with the polarization relationships expected for internal waves, and so in particular having nonzero vertical and density perturbations [88]. Certain key aspects of the flow induced by such a forcing are shown in Fig. 6.

In Fig. 6(a), a snapshot in the $x_{3}-x_{3}$ plane is shown of the pointwise-calculated local (i.e., with no volume-averaging) turbulent kinetic energy dissipation rate $\mathcal{E}_{L}$. The plane is taken from the midpoint of the computational domain at a late time ( $t=150$, scaled in advective time units) after any initial transients associated with the start up of the simulation may be assumed to have passed. A clear layering of the flow is observable, with intense regions of dissipation embedded in much more quiescent regions. This is further evidence that layering appears in a wide range of stratified turbulent flows and also that it is important to be cautious about characterizing flows with volume-averaged quantities as such layering can often occur. 

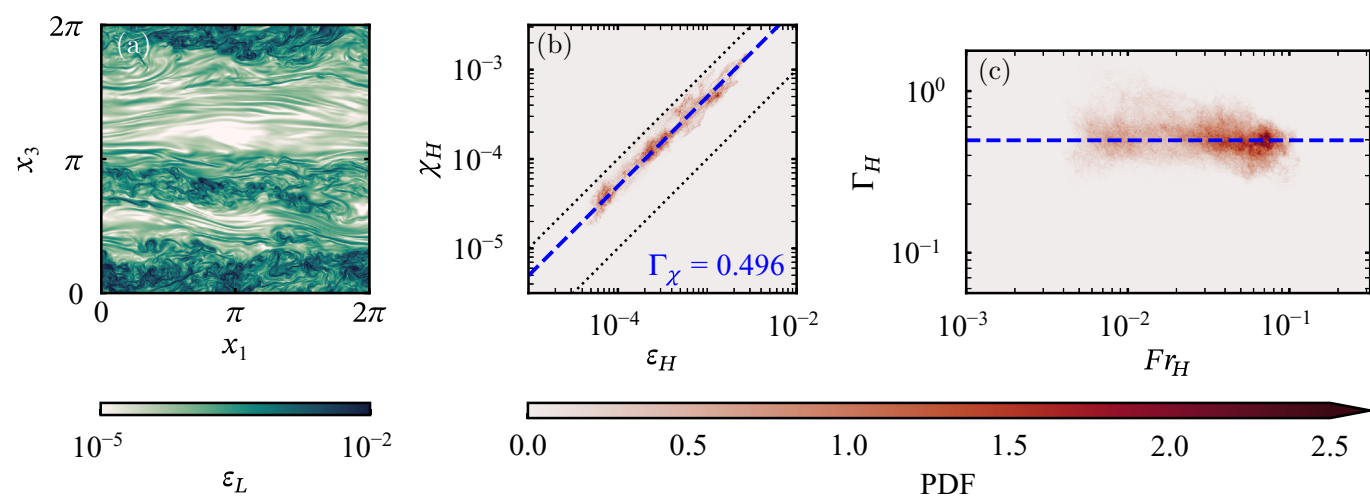

0.0

0.5

1.0

PDF

1.5

2.0

2.5

FIG. 6. In a turbulent flow forced by large-scale internal waves: (a) Snapshot in the $x_{1}-x_{3}$ plane (at the middle of the computational domain) at a late time ( $t=150$, scaled in advective time units) of the pointwise-calculated local turbulent kinetic energy dissipation rate $\mathcal{E}_{L}$. (b) Two-dimensional p.d.f. of horizontally averaged dissipation rates $\mathcal{E}_{H}$ and $\chi_{H}$. The two dotted lines on each panel mark values corresponding to $\Gamma_{H}=0.1$ and $\Gamma_{H}=1$; (c) two-dimensional probability density function (PDF) of $\operatorname{Fr}_{H}$ and $\Gamma_{H}$ calculated from horizontally averaged quantities. Data in (b) and (c) are for a range of nondimensional times $t \geqslant 50$ after initial spin-up, and the blue dashed line marks the volume-averaged value $\Gamma_{\chi}=0.496$. Adapted from and used with permission from Ref. [88], copyright CUP, all rights reserved.

It therefore is natural to consider horizontally averaged quantities as a relatively simple way to identify properties associated with regions of vigorous (and not-so-vigorous) turbulence. In Fig. 6(b), the two-dimensional probability density function (PDF) is plotted of the horizontally averaged kinetic energy dissipation rate $\mathcal{E}_{H}$ and the horizontally averaged buoyancy variance dissipation rate $\chi_{H}$. On this plot, diagonal lines correspond to fixed values of the appropriate turbulent flux coefficient $\Gamma_{H}$ constructed from these horizontally averaged quantities, and for comparison, two dotted lines mark 0.1 and 1 . For this particular flow, $\Gamma_{\chi}=0.496$, the ratio of the volume-averaged $\chi$ and $\mathcal{E}$. The blue dashed line marks the equivalent relationship between the horizontally averaged quantities $\chi_{H}$ and $\mathcal{E}_{H}$. Interestingly, the horizontally averaged data lie quite close to this relation, even though the individual values vary by at least two orders of magnitude.

This property is of course consistent with the "strongly stratified" scaling discussed above, in that it is consistent with $\Gamma_{H} \equiv \chi_{H} / \mathcal{E}_{H}$ being constant, and hence independent of appropriate nondimensional parameters within the flow. This is shown in Fig. 6(c), where the two-dimensional p.d.f. of $\Gamma_{H}$ and $\mathrm{Fr}_{H}$ is plotted for the same times $t>50 . \mathrm{Fr}_{H}$ is the turbulent Froude number constructed with horizontally averaged quantities:

$$
\operatorname{Fr}_{H} \equiv \frac{\mathcal{E}_{H}}{N_{H} \mathcal{K}_{H}^{\prime}},
$$

where the subscript $H$ in the terms on the right-hand side denotes horizontal averaging of pointwise evaluated quantities. (Note this is different from the "horizontal Froude number" briefly mentioned above in the context of the LAST regime.) It is clear that $\Gamma_{H} \sim \operatorname{Fr}_{H}^{0}$ over two orders of magnitude, when $\mathrm{Fr}_{H}$ can be reasonably characterized as being "small."

However, it is important to appreciate that these different values of $\operatorname{Fr}_{H}$ are constructed from within the same flow simulation, which nevertheless exhibits great spatial inhomogeneity and anisotropy, in particular being clearly characterized by layering associated with orders of magnitude variability in dissipation rates. Such variability would be expected to be consistent with a correlated variation in other parameters (such as $\mathrm{Re}_{b}$ ). Therefore, apparent independence of flux coefficient with respect to variations in (small) $\mathrm{Fr}_{T}$ may more broadly be associated with the (relatively large) flux coefficient varying little with respect to any of the parameters. 
For this flow, in either the vigorously turbulent regions, or the essentially quiescent layers, the mixing has similar properties, as quantified by an appropriate definition of the flux coefficient. Indeed the mixing throughout the flow appears to be highly "efficient," suggesting that the mixing induced by such internal-wave-driven flows may typically have a high (and hence efficient) value of flux coefficient. Somewhat smaller values $\left(\Gamma_{\chi} \simeq 0.37\right)$ were associated with purely horizontal forcing, suggesting that the "breaking" of internal waves may well lead to more efficient mixing, perhaps associated with being "convective" in character [90,91]. Also, it could be argued that the differences observed previously in this (apparently) asymptotic value at low $\mathrm{Fr}_{T}$ may well be associated with particular aspects of the large-scale forcing.

The fundamental quantity of interest, the actual absolute amount of diapycnal mixing, will be dominated by the more turbulent regions (as is apparent from the definition of $\kappa_{T}$ multiplying the flux coefficient by $\mathrm{Re}_{b}$ ). There are curious, and still unexplained observations, that these clearly apparent "layers" in dissipation rate do not correlate with local changes in the background shear and stratification, and that the specific value of the flux coefficient is broadly similar in both the turbulent and quiescent regions of the flow. Nevertheless, that stratified turbulence is generically layered is a viewpoint which is gaining more and more supporting evidence.

\section{VI. "CONCLUSION": IS ANYTHING CERTAIN?}

In this article, I have attempted both to convey some of the interesting advances in the last few years and also to highlight remaining areas of profound uncertainty in understanding and modeling (irreversible) turbulent mixing in stratified flows. As discussed in Sec. II, a fundamental challenge in stratified mixing has been, for several decades, modeling of a (turbulent) eddy diffusivity for the density (or "heat"), i.e., $\kappa_{T}$. Although in fact a subsidiary, derived quantity, a large amount of research effort has actually been devoted to the parametrization of some appropriate definition of a turbulent flux coefficient $\Gamma$. More specifically, there have been many studies investigating whether anything generic can be said about the functional form of $\Gamma\left(\operatorname{Re}_{b}, \mathrm{Ri}, \mathrm{Fr}_{T}, \operatorname{Pr}\right)$, where arguments have often been presented that a particular nondimensional parameter is physically meaningful.

Of course, there are (at least) two very reasonable and fundamental criticisms of the underlying philosophy identifying the modeling of a flux coefficient as the most important open question requiring an answer. First, it is self-evident that modeling scalar transport in a turbulent flow using such a flux-gradient model involving an eddy diffusivity $\kappa_{T}$ is generically likely to be inaccurate. Furthermore, focus of attention on flux coefficients has the implicit assumption that it is sensible to attempt to describe irreversible mixing rates (quantified through $\mathcal{B}, \mathcal{M}$, or $\chi$, averaged in some way) in terms of the turbulent dissipation rate $\mathcal{E}$, once again averaged in some way. That it is appropriate to model stratified mixing in terms of such a flux coefficient $\Gamma$ "taxation rate" relating mixing rates to the turbulent kinetic energy dissipation rate is really a rather strong, and indeed often highly inappropriate, assumption. Also, focus on $\Gamma$ unfortunately obscures the fact that it is really the diapycnal scalar transport which is the process of fundamental interest.

Nevertheless, even accepting these criticisms, and carrying on regardless in an attempt to construct a fluid-dynamically justified functional form for some definition of flux coefficient $\Gamma$ (or other measures of mixing efficiency), strong evidence is emerging that it is extremely difficult to say anything generic about the parameterization of stratified mixing. The influential, indeed seductively appealing hypothesis that $\Gamma\left(\operatorname{Re}_{b}\right)$ in some nontrivial way $[9,37,38]$ appears (at the moment) to be inconsistent with the evidence of at least one highly idealized flow. This stratified shear flow is forced (by design) to be statistically steady and spatially homogeneous [29], and (perhaps fortuitously) characterized by essentially constant values of the other (assumed) key parameters, thus isolating the (non-)effects of variation in $\mathrm{Re}_{b}$.

However, it cannot be said that flux coefficients, or equivalently "mixing efficiencies," never depend on $\mathrm{Re}_{b}$. Both observations and "snapshots" of the highly time-dependent mixing induced by the breakdown of primary KHI does indeed exhibit such a dependence, consistent with the characteristic scaling of $\Gamma \sim \operatorname{Re}_{b}^{-1 / 2}$ at high $\operatorname{Re}_{b}$ previously assumed. However, it is not yet clear 
how important the time dependence of this flow is, i.e., it is an open question as to just how much "history matters," associated with the vigorous primary, and perhaps optimal "overturning" [51].

Indeed, the mixing dichotomy between more efficient "overturning" and less efficient "scouring" appears to be a useful way to think about stratified mixing, at least in the situation of shear-driven mixing. "Scouring," associated with sharper and stronger density interfaces and the breakdown of the other classical shear instability HWI, appears to be associated with at least a "kind of equilibrium," and so longer-lived periods of turbulent mixing. Just as in wall-forced stratified plane Couette flow, for such relatively long-lasting turbulent mixing, it seems reasonable to think of the flow as being "weakly stratified," in the specific sense that $\mathrm{Ri} \sim \mathrm{Ri}_{f} \lesssim 0.2$ and so $\operatorname{Pr}_{T} \sim O(1)$, implying momentum and scalar are "mixed" similarly.

This observation therefore naturally motivates the search for flows which can be characterized as being both strongly stratified (e.g., with $\mathrm{Fr}_{T} \ll 1$ ) and vigorously turbulent (e.g., with $\mathrm{Re}_{b} \gg 1$ ). This search must always keep in mind two major potentially misleading phenomena. The first is that it is important to monitor whether there is correlation between parameters, as generically it is not yet established that real turbulent flows can access every part of the multidimensional parameter space. Second, and perhaps more importantly, there is mounting evidence, in simulation [85], experiment [75], and even observation [91], that spatiotemporal variability is a central, perhaps even defining characteristic of stratified turbulent mixing processes. This mounting evidence points clearly toward the fact that the structure of turbulent stratified flow states exhibits significant spatiotemporal variability. Just to take one example, there is a lot of evidence that "layering is generic," in the sense that, in a wide range of circumstances, approximately constant density gradients are broken down by stratified turbulence spontaneously into a "staircase" of relatively well-mixed and deep "layers," separated by relatively thin "interfaces" with increased density gradient [3].

Although spatiotemporal variability in the flow structures has been widely identified and studied in idealized simulations and laboratory experiments, as already noted the larger-scale flow forcing has usually been quite simple. As discussed above, there have been studies which have been forced, essentially quasisteadily in time either at a boundary (for example in stratified Taylor-Couette flow or plane Couette flow), or through artificial body forces in a numerical simulation. There have also been many unforced (at least after time $t=0$ ) simulations and experiments, where the initial value problem of the subsequent flow evolution from an initial, deliberately constructed, "background flow" is considered. In real geophysical flows, it seems much more likely that the large-scale forcing of the small-scale turbulence (and ensuing mixing) will itself be spatiotemporally variable. For example, Ref. [92] investigated time-periodically varying shear flows as a model of the interaction between KHI and propagating internal waves. It is clearly very important to investigate further whether such spatiotemporal variability in forcing leads to qualitatively different behavior, particularly at sufficiently high Reynolds number for vigorous turbulent mixing to occur. Put simply, has traditional focus on the simpler end member classes (of either quasisteady forcing or "free" initial value problems) thrown the geophysically relevant mixing "baby" out with the overidealized "bathwater"?

Indeed, care must be taken to determine whether a particular value of some quantity (such as a flux coefficient) of interest, averaged in time and/or space, is made up of a combination of qualitatively different "patches," "layers," or "regions." A particular open question of great interest is whether a flow which appears to be on average both strongly stratified and vigorously turbulent typically contains highly spatiotemporally intermittent patches of vigorous, yet locally weakly stratified turbulence embedded within largely quiescent regions of exceptionally strongly stratified fluid [85]. Studying such a "patchy" and/or "layer-interface" flow requires a robust method to identify and characterize these "structures" underlying the flow "statistics," where it has long been known that there appears to be at least an imprint of inhomogeneity and anisotropy [80,93]. It also inevitably calls into question how the various physical quantities (for example $N, S, \mathcal{E}$, $\chi$, etc.) are actually averaged for input into the different nondimensional parameters used in modeling. Just to mention one issue, the definition (13) of the Ozmidov length scale $L_{O}$ must be treated with caution. A reasonable interpretation is that vigorous turbulence (with a relatively vertically 
localized dissipation rate $\mathcal{E}$ ) is embedded within a background, relatively more vertically extended stratification, with characteristic buoyancy frequency $N$. The relative vertical extents over which $\mathcal{E}$ and $N$ are calculated must be clearly understood for appropriate calculation and interpretation of $L_{O}$.

With the explosion in the development of a huge variety of data-driven techniques, stratified turbulent mixing seems a research area ripe for the application of artificial intelligence, and there have already been some promising first steps in this direction $[85,94,95]$. Such data-driven approaches, when allied with the deep physical insight garnered over decades of fluid dynamical research, is perhaps the way forward to unravel at last some aspects of the fascinating and crucially important mystery that is turbulent mixing in stratified fluids.

\section{ACKNOWLEDGMENTS}

My research has been inspired and mentored over three decades by my Ph.D. supervisor, Paul Linden, and my postdoctoral principal investigator, Dick Peltier. I have been exceptionally lucky to work with many brilliant and hard-working students, postdoctoral fellows, and collaborators from all over the world. Special thanks go to Chris Howland, Ali Mashayek, Gavin Portwood, and Hesam Salehipour for assistance with the figures in this paper and also the two reviewers of this manuscript, whose constructive comments have proved very useful and insightful. I wish to acknowledge both the financial support of EPSRC and the contributions of all those involved in the 2013-2018 Programme Grant EP/K034529/1 entitled "Mathematical Underpinnings of Stratified Turbulence." I am also deeply grateful to the National Science Foundation for the sustained support of the Summer Study Program in GFD at Woods Hole Oceanographic Institution. In particular, I thank all the participants in the 2019 program on "Stratified Turbulence and Ocean Mixing Processes." I also thank all the participants in the Les Houches Conference "Environmental Fluid Dynamics: Confronting Grand Challenges" in January 2019, where I gave a preliminary version of the underlying presentation. Finally, I wish to express my deep appreciation to the APS Division of Fluid Dynamics for the honour and opportunity to share my thoughts on this problem at the 72nd Annual Meeting of the Division, held in Seattle in November 2019.

[1] M. C. Gregg, E. A. D’Asaro, J. J. Riley, and E. Kunze, Mixing efficiency in the ocean, Annu. Rev. Mar. Sci. 10, 443 (2018).

[2] J. A. MacKinnon, Z. X. Zhao, C. Whalen, A. F. Waterhouse, D. S. Trossman, O. M. Sun, L. St Laurent, H. L. Simmons, K. Polzin, R. Pinkel, A. Pickering, N. J. Norton, J. D. Nash, R. Musgrave, L. M. Merchant, A. V. Melet, B. Mater, S. Legg, W. G. Large, E. Kunze, J. M. Klymak, M. Jochum, S. R. Jayne, R. W. Hallberg, S. M. Griffies, S. Diggs, G. Danabasoglu, E. P. Chassignet, M. C. Buijsman, F. O. Bryan, B. P. Briegleb, A. Barna, B. K. Arbic, J. K. Ansong, and M. H. Alford, Climate process team on internal wave-driven ocean mixing, Bull. Am. Meteorol. Soc. 98, 2429 (2017).

[3] C. P. Caulfield, Layering, instabilities and mixing in turbulent stratified flows, Annu. Rev. Fluid Mech. 53, 113 (2021).

[4] R. W. Schmitt, Double diffusion in oceanography, Annu. Rev. Fluid Mech. 26, 255 (1994).

[5] R. Tailleux, On the energetics of stratified turbulent mixing, irreversible thermodynamics, Boussinesq models and the ocean heat engine controversy, J. Fluid Mech. 638, 339 (2009).

[6] R. Tailleux, Available potential energy density for a multicomponent Boussinesq fluid with arbitrary nonlinear equation of state, J. Fluid Mech. 735, 499 (2013).

[7] P. F. Linden, Mixing in stratified fluids, Geophys. Astrophys. Fluid Dyn. 13, 431 (1979).

[8] H. J. S. Fernando, Turbulent mixing in stratified fluids, Annu. Rev. Fluid Mech. 23, 455 (1991).

[9] G. N. Ivey, K. B. Winters, and J. R. Koseff, Density stratification, turbulence, but how much mixing, Annu. Rev. Fluid Mech. 40, 169 (2008). 
[10] T. R. Osborn, Estimates of the local-rate of vertical diffusion from dissipation measurements, J. Phys. Oceanogr. 10, 83 (1980).

[11] A. F. Waterhouse, J. A. MacKinnon, J. D. Nash, M. H. Alford, E. Kunze, H. L. Simmons, K. L. Polzin, L. C. St Laurent, O. M. Sun, R. Pinkel, L. D. Talley, C. B. Whalen, T. N. Huussen, G. S. Carter, I. Fer, S. Waterman, A. C. N. Garabato, T. B. Sanford, and C. M. Lee, Global patterns for diapycnal mixing from measurements of the turbulent dissipation rate, J. Phys. Oceanogr. 44, 1854 (2014).

[12] M. S. Davies Wykes and S. B. Dalziel, Efficient mixing in stratified flows: Experimental study of a Rayleigh-Taylor unstable interface within an otherwise stable stratification, J. Fluid Mech. 756, 1027 (2014).

[13] E. N. Lorenz, Available potential energy and the maintenance of the general circulation, Tellus 7, 157 (1955).

[14] K. B. Winters, P. N. Lombard, J. J. Riley, and E. A. D'Asaro, Available potential-energy and mixing in density-stratified fluids, J. Fluid Mech. 289, 115 (1995).

[15] K. B. Winters and E. A. D'Asaro, Diascalar flux and the rate of fluid mixing, J. Fluid Mech. 317, 179 (1996).

[16] H. Salehipour and W. R. Peltier, Diapycnal diffusivity, turbulent Prandtl number and mixing efficiency in Boussinesq stratified turbulence, J. Fluid Mech. 775, 464 (2015).

[17] C. P. Caulfield and W. R. Peltier, The anatomy of the mixing transition in homogeneous and stratified free shear layers, J. Fluid Mech. 413, 1 (2000).

[18] W. R. Peltier and C. P. Caulfield, Mixing efficiency in stratified shear flows, Annu. Rev. Fluid Mech. 35, 135 (2003).

[19] E. Villermaux, Mixing versus stirring, Annu. Rev. Fluid Mech. 51, 245 (2019).

[20] R. S. Arthur, S. K. Venayagamoorthy, J. R. Koseff, and O. B. Fringer, How we compute $N$ matters to estimates of mixing in stratified flows, J. Fluid Mech. 831, R2 (2017).

[21] D. G. Andrews, A note on potential-energy density in a stratified compressible fluid, J. Fluid Mech. 107, 227 (1981).

[22] D. Holliday and M. E. McIntyre, On potential-energy density in a incompressible stratified fluid, J. Fluid Mech. 107, 221 (1981).

[23] A. Scotti and B. White, Diagnosing mixing in stratified turbulent flows with a locally defined available potential energy, J. Fluid Mech. 740, 114 (2014).

[24] K. R. Sreenivasan, Turbulent mixing: A perspective, Proc. Nat. Acad. Sci. USA 116, 18175 (2019).

[25] T. R. Osborn and C. S. Cox, Oceanic fine structure, Geophys. Fluid Dyn. 3, 321 (1972).

[26] G. N. Ivey, C. E. Bluteau, and N. L. Jones, Quantifying diapycnal mixing in an energetic ocean, J. Geophys. Res.-Oceans 123, 346 (2018).

[27] J. R. Taylor, S. M. de Bruyn Kops, C. P. Caulfield, and P. F. Linden, Testing the assumptions underlying ocean mixing methodologies using direct numerical simulations, J. Phys. Oceanogr. 49, 2761 (2019).

[28] H. Salehipour, C. P. Caulfield, and W. R. Peltier, Turbulent mixing due to the Holmboe wave instability at high Reynolds number, J. Fluid Mech. 803, 591 (2016).

[29] G. D. Portwood, S. M. de Bruyn Kops, and C. P. Caulfield, Asymptotic Dynamics of High Dynamic Range Stratified Turbulence, Phys. Rev. Lett. 122, 194504 (2019).

[30] J. W. Miles, On the stability of heterogeneous shear flows, J. Fluid Mech. 10, 496 (1961).

[31] L. N. Howard, Note on a paper of John W. Miles, J. Fluid Mech. 10, 509 (1961).

[32] J. S. Turner, Buoyancy Effects in Fluids (Cambridge University Press, Cambridge, UK, 1973).

[33] A. Maffioli, G. Brethouwer, and E. Lindborg, Mixing efficiency in stratified turbulence, J. Fluid Mech. 794, R3 (2016).

[34] B. D. Mater and S. K. Venayagamoorthy, A unifying framework for parametrizing stably stratified shearflow turbulence, Phys. Fluids 26, 036601 (2014).

[35] B. D. Mater and S. K. Venayagamoorthy, The quest for an unambiguous parametrization of mixing efficiency in stably stratified geophysical flows, Geophys. Res. Lett. 41, 4646 (2014).

[36] D. Lucas and C. P. Caulfield, Irreversible mixing by unstable periodic orbits in buoyancy dominated stratified turbulence, J. Fluid Mech. 832, R1 (2017). 
[37] L. H. Shih, J. R. Koseff, G. N. Ivey, and J. H. Ferziger, Parameterization of turbulent fluxes and scales using homogeneous sheared stably stratified turbulence simulations, J. Fluid Mech. 525, 193 (2005).

[38] S. G. Monismith, J. R. Koseff, and B. White, Mixing efficiency in the presence of stratification: When is it constant, Geophys. Res. Lett. 45, 5627 (2018).

[39] Q. Zhou, J. R. Taylor, and C. P. Caulfield, Self-similar mixing in stratified plane Couette flow for varying Prandtl number, J. Fluid Mech. 820, 86 (2017).

[40] E. Deusebio, C. P. Caulfield, and J. R. Taylor, The intermittency boundary in stratified plane Couette flow, J. Fluid Mech. 781, 298 (2015).

[41] A. Scotti and B. White, The mixing efficiency of stratified turbulent boundary layers, J. Phys. Oceanogr. 46, 181 (2016).

[42] O. Flores and J. J. Riley, Analysis of turbulence collapse in the stably stratified surface layer using direct numerical simulation, Bound.-Layer Meteorol. 139, 241 (2011).

[43] A. K. Kaminski, C. P. Caulfield, and J. R. Taylor, Transient growth in strongly stratified shear layers, J. Fluid Mech. 758, R4 (2014).

[44] C. J. Howland, J. R. Taylor, and C. P. Caulfield, Testing linear marginal stability in stratified shear layers, J. Fluid Mech. 839, R4 (2018).

[45] A. K. Kaminski and W. D. Smyth, Stratified shear instability in a field of pre-existing turbulence, J. Fluid Mech. 862, 639 (2019).

[46] A. K. Kaminski, C. P. Caulfield, and J. R. Taylor, Nonlinear evolution of linear optimal perturbations of strongly stratified shear layers, J. Fluid Mech. 825, 213 (2017).

[47] J. P. Parker, C. P. Caulfield, and R. R. Kerswell, Kelvin-Helmholtz billows above Richardson number 1/4, J. Fluid Mech. 879, R1 (2019).

[48] J. P. Parker, C. P. Caulfield, and R. R. Kerswell, The viscous Holmboe instability for smooth shear and density profiles, J. Fluid Mech. 896, A14 (2020).

[49] T. S. Eaves and C. P. Caulfield, Multiple instability of layered stratified plane Couette flow, J. Fluid Mech. 813, 250 (2017).

[50] A. Mashayek, C. P. Caulfield, and W. R. Peltier, Time-dependent, non-monotonic mixing in stratified shear flows: Implications for oceanographic estimates of buoyancy flux? J. Fluid Mech. 736, 570 (2013).

[51] A. Mashayek, C. P. Caulfield, and W. R. Peltier, Role of overturns in optimal mixing in stratified mixing layers, J. Fluid Mech. 826, 522 (2017).

[52] W. D. Smyth, G. P. Klaassen, and W. R. Peltier, Finite amplitude Holmboe waves, Geophys. Astrophys. Fluid Dyn. 43, 181 (1988).

[53] A. W. Woods, C. P. Caulfield, J. R. Landel, and A. Kuesters, Non-invasive mixing across a density interface in a turbulent Taylor-Couette flow, J. Fluid Mech. 663, 347 (2010).

[54] J. R. Taylor and Q. Zhou, A multi-parameter criterion for layer formation in a stratified shear flow using sorted buoyancy coordinates, J. Fluid Mech. 823, R5 (2017).

[55] Q. Zhou, J. R. Taylor, C. P. Caulfield, and P. F. Linden, Diapycnal mixing in layered stratified plane Couette flow quantified in a tracer-based coordinate, J. Fluid Mech. 821, 198 (2017).

[56] A. Mashayek and W. R. Peltier, The 'zoo' of secondary instabilities precursory to stratified shear flow transition. Part 1: Shear aligned convection, pairing and braid instabilities, J. Fluid Mech. 708, 5 (2012).

[57] A. Mashayek and W. R. Peltier, The 'zoo' of secondary instabilities precursory to stratified shear flow transition. Part 2: The influence of stratification, J. Fluid Mech. 708, 45 (2012).

[58] J. Holmboe, On the behavior of symmetric waves in stratified shear layers, Geofys. Publ. 24, 67 (1962).

[59] A. Lefauve, J. L. Partridge, Q. Zhou, S. B. Dalziel, C. P. Caulfield, and P. F. Linden, The structure and origin of confined Holmboe waves, J. Fluid Mech. 848, 508 (2018).

[60] E. W. Tedford, R. Peters, and G. A. Lawrence, Symmetric Holmboe instabilities in a laboratory exchange flow, J. Fluid Mech. 636, 137 (2009).

[61] G. A. Lawrence, F. K. Browand, and L. G. Redekopp, The stability of a sheared density interface, Phys. Fluids A: Fluid Dyn. 3, 2360 (1991).

[62] H. Salehipour, W. R. Peltier, and C. P. Caulfield, Self-organised criticality of turbulence in strongly stratified mixing layers, J. Fluid Mech. 856, 228 (2018). 
[63] W. D. Smyth, J. D. Nash, and J. N. Moum, Self-organized criticality in geophysical turbulence, Sci. Rep. 9, 3747 (2019).

[64] A. Mashayek and W. R. Peltier, Shear-induced mixing in geophysical flows: Does the route to turbulence matter to its efficiency? J. Fluid Mech. 725, 216 (2013).

[65] W. D. Smyth, J. N. Moum, and D. R. Caldwell, The efficiency of mixing in turbulent patches: Inferences from direct simulations and microstructure observations, J. Phys. Oceanogr. 31, 1969 (2001).

[66] D. Bouffard and L. Boegman, A diapycnal diffusivity model for stratified environmental flows, Dyn. Atmos. Oceans 61-62, 14 (2013).

[67] A. Mashayek, H. Salehipour, D. Bouffard, C. P. Caulfield, R. Ferrari, M. Nikurashin, W. R. Peltier, and W. D. Smyth, Efficiency of turbulent mixing in the abyssal ocean circulation, Geophys. Res. Lett. 44, 6296 (2017).

[68] A. Deloncle, J.-M. Chomaz, and P. Billant, Three-dimensional stability of a horizontally sheared flow in a stably stratified fluid, J. Fluid Mech. 570, 297 (2007).

[69] D. Lucas, C. P. Caulfield, and R. R. Kerswell, Layer formation in horizontally forced stratified turbulence: Connecting exact coherent structures to linear instabilities, J. Fluid Mech. 832, 409 (2017).

[70] P. Billant and J.-M. Chomaz, Experimental evidence for a new instability of a vertical columnar vortex pair in a strongly stratified fluid, J. Fluid Mech. 418, 167 (2000).

[71] P. Billant and J.-M. Chomaz, Theoretical analysis of the zigzag instability of a vertical columnar vortex pair in a strongly stratified fluid, J. Fluid Mech. 419, 29 (2000).

[72] M. A. Deloncle, P. Billant, and J.-M. Chomaz, Nonlinear evolution of the zigzag instability in stratified fluids: A shortcut on the route to dissipation, J. Fluid Mech. 599, 229 (2008).

[73] M. L. Waite and P. Bartello, Instability and breakdown of a vertical vortex pair in a strongly stratified fluid, J. Fluid Mech. 517, 281 (2008).

[74] P. Augier and P. Billant, Onset of secondary instabilities on the zigzag instability in stratified fluids, J. Fluid Mech. 682, 120 (2011).

[75] R. L. F. Oglethorpe, C. P. Caulfield, and A. W. Woods, Spontaneous layering in stratified turbulent TaylorCouette flow, J. Fluid Mech. 721, R3 (2013).

[76] M. J. Molemaker, J. C. McWilliams, and I. Yavneh, Instability and Equilibration of Centrifugally Stable Stratified Taylor-Couette Flow, Phys. Rev. Lett. 86, 5270 (2001).

[77] M. Le Bars and P. Le Gal, Experimental Analysis of the Stratorotational Instability in a Cylindrical Couette Flow, Phys. Rev. Lett. 99, 064502 (2007).

[78] K. N. Singh, Instability and mixing in a turbulent stratified taylor-couette flow, Ph.D. thesis, University of Cambridge, 2019.

[79] D. Petrolo and S. Longo, Buoyancy transfer in a two-layer system in steady state. Experiments in a Taylor-Couette cell, J. Fluid Mech. 896, A27 (2020).

[80] M. Falder, N. J. White, and C. P. Caulfield, Seismic imaging of rapid onset of stratified turbulence in the South Atlantic Ocean, J. Phys. Oceanogr. 46, 1023 (2016).

[81] P. Billant and J.-M. Chomaz, Self-similarity of strongly stratified inviscid flows, Phys. Fluids 13, 1645 (2001).

[82] G. Brethouwer, P. Billant, E. Lindborg, and J.-M. Chomaz, Scaling analysis and simulation of strongly stratified turbulent flows, J. Fluid Mech. 1585, 343 (2007).

[83] P. Bartello and S. M. Tobias, Sensitivity of stratified turbulence to the buoyancy Reynolds number, J. Fluid Mech. 725, 1 (2013).

[84] Q. Zhou and P. J. Diamessis, Large-scale characteristics of stratified wake turbulence at varying Reynolds number, Phys. Rev. Fluids 4, 084802 (2019).

[85] G. D. Portwood, S. M. de Bruyn Kops, J. R. Taylor, H. Salehipour, and C. P. Caulfield, Robust identification of dynamically distinct regions in stratified turbulence, J. Fluid Mech. 807, R2 (2016).

[86] M. Wells, C. Cenedese, and C. P. Caulfield, The relationship between flux coefficient and entrainment ratio in density currents, J. Phys. Oceanogr. 40, 2713 (2010).

[87] A. Garanaik and S. K. Venayagamoorthy, On the inference of the state of turbulence and mixing efficiency in stably stratified flows, J. Fluid Mech. 867, 323 (2019). 
[88] C. J. Howland, J. R. Taylor, and C. P. Caulfield, Mixing in forced stratified turbulence and its dependence on large-scale forcing, J. Fluid Mech. 898, A7 (2020).

[89] A. Maffioli, Vertical spectra of stratified turbulence at large horizontal scales, Phys. Rev. Fluids 2, 104802 (2017).

[90] M. S. Davies Wykes, G. O. Hughes, and S. B. Dalziel, On the meaning of mixing efficiency for buoyancydriven mixing in stratified turbulent flows, J. Fluid Mech. 781, 261 (2015).

[91] T. Ijichi, L. St. Laurent, K. L. Polzin, and J. M. Toole, How variable is mixing efficiency in the abyss? Geophys. Res. Lett. 47, e2019GL086813 (2020).

[92] R. Inoue and W. D. Smyth, Efficiency of mixing forced by unsteady shear flow, J. Phys. Oceanogr. 39, 1150 (2009).

[93] H. Yamazaki and R. Lueck, Why oceanic dissipation rates are not lognormal, J. Phys. Oceanogr. 20, 1907 (1990).

[94] H. Salehipour and W. R. Peltier, Deep learning of mixing by two 'atoms' of stratified turbulence, J. Fluid Mech. 861, R4 (2019).

[95] T. Bolton and L. Zanna, Applications of deep learning to ocean data inference and subgrid parameterization, J. Adv. Mod. Earth Syst. 11, 376 (2019). 